\title{
OPEN Targeted deletion of the RNA-binding protein Caprin1 leads to progressive hearing loss and impairs recovery from noise exposure in mice
}

\author{
Lisa S. Nolan ${ }^{1,2}$, Jing Chen ${ }^{2}$, Ana-Cláudia Gonçalves ${ }^{1}$, Anwen Bullen ${ }^{1}$, Emily R. Towers ${ }^{1}$, \\ Karen P. Steel ${ }^{2}$, Sally J. Dawson ${ }^{1,3 凶}$ \& Jonathan E. Gale ${ }^{1,3 \bowtie}$
}

Cell cycle associated protein 1 (Caprin1) is an RNA-binding protein that can regulate the cellular post-transcriptional response to stress. It is a component of both stress granules and neuronal RNA granules and is implicated in neurodegenerative disease, synaptic plasticity and long-term memory formation. Our previous work suggested that Caprin1 also plays a role in the response of the cochlea to stress. Here, targeted inner ear-deletion of Caprin1 in mice leads to an early onset, progressive hearing loss. Auditory brainstem responses from Caprin1-deficient mice show reduced thresholds, with a significant reduction in wave-I amplitudes compared to wildtype. Whilst hair cell structure and numbers were normal, the inner hair cell-spiral ganglion neuron (IHC-SGN) synapse revealed abnormally large post-synaptic GluA2 receptor puncta, a defect consistent with the observed wave-I reduction. Unlike wildtype mice, mild-noise-induced hearing threshold shifts in Caprin1-deficient mice did not recover. Oxidative stress triggered TIA-1/HuR-positive stress granule formation in ex-vivo cochlear explants from Caprin1-deficient mice, showing that stress granules could still be induced. Taken together, these findings suggest that Caprin1 plays a key role in maintenance of auditory function, where it regulates the normal status of the IHC-SGN synapse.

Cell cycle associated protein 1 (Caprin1; also known as RNG105) is a RNA-binding protein originally identified as a promoter of cell proliferation ${ }^{1,2}$. Subsequently, it has been shown to be a core nucleating component of stress granules ${ }^{3,4}$ and also to regulate local RNA translation in neuronal RNA granules ${ }^{5-7}$. Stress granules are concentrated aggregates of RNA binding proteins and RNA which form by liquid-liquid phase separation under certain types of stress ${ }^{4,8,9}$. Caprin 1 plays a key role in promoting this condensation mechanism by competing with USP10 for binding to G3BP $1^{4,10}$. In a highly dynamic mechanism stress granules recruit specific RNAs, inhibiting translation of these RNAs, either storing or degrading them during cellular stress via interactions with processing bodies. In recent years many components of stress granules have been identified and the mechanism regulating their formation has been elucidated yet their precise function is still unclear. It is suggested that stress granules are a pro-survival mechanism, regulating translation of mRNAs and coordinating cell signaling during stress thereby contributing to cellular homeostasis ${ }^{11,12}$.

In contrast to their suggested role in protecting cells during cellular stress, stress granules have been linked with various pathologies including cancer, viral infections and neurodegeneration ${ }^{11,13,14}$. In cancer, assembly of stress granules is associated with resistance to chemotherapy and metastasis ${ }^{15}$. During viral infection many different viruses have been shown to use various strategies to inhibit stress granule formation designed to avoid stress granule-mediated stalling of viral RNA translation ${ }^{9}$. Mutation of stress granule components including TDP-43, TIA-1 and FUS is associated with neurodegenerative diseases such as amyotrophic lateral sclerosis and core stress granule components are localized to pathological aggregates in Alzheimer's disease and Huntington's disease $\mathrm{e}^{11,13,14}$. Accumulation of such aberrant or persistent stress granules in pathological aggregates associated

${ }^{1}$ UCL Ear Institute, 332 Gray's Inn Road, London WC1X 8EE, UK. ${ }^{2}$ Wolfson Centre for Age-Related Diseases, King's College London, Guy's Campus, London SE1 1UL, UK. ${ }^{3}$ These authors contributed equally: Sally J. Dawson and Jonathan E. Gale. ${ }^{\circledR}$ email: sally.dawson@ucl.ac.uk; j.e.gale@ucl.ac.uk 
with neurodegeneration has been attributed to the dysregulation of stress granule formation/disassembly and has been implicated in age-related disease ${ }^{14}$.

Previously, we have identified a role for Caprin1 and stress granules in the response to cochlear stress ${ }^{16,17}$. The process of hearing sound is a very sensitive and sophisticated process that itself generates various types of intrinsic cellular stress: mechanical, ionic, oxidative, and synaptic ${ }^{18,19}$. The cochlea is also subject throughout its life to various kinds of additional extrinsic stresses most notably as a result of damage from environmental agents such as noise and ototoxic drugs (e.g. aminoglycoside antibiotics and cisplatin $)^{20,21}$. All of these mechanisms can contribute to hearing loss and their effects are cumulative resulting in a common age-related decline in auditory function $^{22}$. Caprin1-positive stress granules form in sensory hair cells of the mammalian inner ear in response to aminoglycoside antibiotics and pharmacological manipulation of stress granule formation can protect sensory hair cells from aminoglycoside-induced ototoxicity ${ }^{16}$. These data suggest Caprin 1 and stress granules play an important role in auditory protection during cellular stress.

Since, Caprin1 homozygote knockout mice exhibit neonatal lethality due to respiratory failure we created an inner ear conditional Caprin1 knockout mouse (Caprin1 cKO), driven by the Sox10-Cre allele ${ }^{23}$ in which to study the role of Caprin 1 and stress granules in auditory protection during cellular stress. We show that Caprin1 deficient mice still form stress granules in response to arsenite treatment suggesting Caprin1 is not necessary for stress granule formation. However, mice lacking Caprin1 in the inner ear exhibit an early-onset hearing loss that progresses with age and an inability to recover from the effects of mild-noise exposure unlike wildtype mice. Furthermore, we provide evidence that loss of the Caprin1 protein in mice leads to a defect in the IHCSGN synapse structure and function, evidenced by a reduction in auditory brain stem response (ABR) wave-I amplitudes and changes in the morphology of the post-synaptic puncta, observed by labelling the GluA2 subunit of the post-synaptic AMPA-type ionotropic glutamate receptors. Our results have implications for our understanding of the role of RNA granule proteins and their role in determining how the auditory system responds and recovers from damage.

\section{Results}

Generation of Caprin1 deficient mice. Mice carrying a germline knockout for the Caprin1 gene are neonatal lethal ${ }^{5}$. Therefore, to understand the role of Caprin 1 in the auditory system we undertook a conditional knockout approach using a Sox10-cre driver specific to inner ear and craniofacial neural crest-derived tissues ${ }^{24}$. Mice carrying a conditional allele for Caprin1 were generated at the Wellcome Sanger Institute, U.K. and crossed with mice carrying the Sox10-cre transgene to generate conditional knockout Caprin1 ${ }^{\text {tm } 3 d / t m 3 d}$ (Caprin1 cKO) mice (Fig. 1A). In contrast to the systemic knockout, Caprin $1^{t m 3 d / t m 3 d}$ mice were viable and survived to adulthood. Quantitative PCR confirmed significant knockdown of the Caprin1 mRNA transcript in the brain of Caprin $1^{t m 3 d / t m 3 d}$ mice compared to control mice $(\mathrm{p}=2 \mathrm{E}-06)$, with heterozygote mice having intermediate levels of expression (Fig. 1B). Caprin1 protein expression was compared in the cochleae of Caprin $1^{\text {tm } 3 d / t m 3 d}$ and wildtype mice using immunofluorescence (Fig. 1C-H). In wildtype mice at P28, Caprin1 expression appears relatively ubiquitous as described for other tissues ${ }^{3}$. However, increased immunoreactivity was observed in inner hair cells (IHC) within the organ of Corti (Fig. 1C, arrowhead), and in the spiral ganglion (SG) (Fig. 1C,E,G). Labelling of the Deiter's cells that cradle the outer hair cells (OHCs) was also apparent (arrow in Fig. 1E). In the cochlea of the Caprin1 cKO mice, Caprin 1 immunoreactivity was much reduced (Fig. 1D) and the reduction was particularly notable in the IHC region. A very small number of cells appeared to escape the Cre recombination and continued to express Caprin1 protein (see SG in Fig. 1D). The gross anatomy and overall structure of the cochlea was not altered in the $\mathrm{CKO}$ mice with three rows of outer hair cells and a single row of inner hair cells present in mutant and wildtype mice.

Caprin1-deficient mice exhibit early-onset progressive hearing loss. To assess the effect of Caprin1-deletion on hearing function auditory brainstem response (ABR) recordings were performed at successive time-points in a longitudinal hearing study out to postnatal day (P)210 (Fig. 2A). At P28, the earliest time-point assessed, hearing thresholds in Caprin $1^{\text {tm } 3 d / t m 3 d}$ mice were already significantly elevated across most frequencies compared to wildtype controls (Fig. 2A). With increasing age loss of auditory sensitivity in Caprin $1^{t m 3 d / t m 3 d}$ mice progressed significantly in the mid to high-frequencies $(18-24 \mathrm{kHz})$, such that by $\mathrm{P} 210$ little recordable hearing could be detected at frequencies above $18 \mathrm{kHz}$ (Fig. 2A). In comparison, a slower loss of auditory sensitivity was observed in the low to mid-frequency range (Fig. $2 \mathrm{~A} \mathrm{e.g.} 12 \mathrm{kHz}$ ). Hearing thresholds in heterozygous mice $\left(\right.$ Caprin $1^{+/ t m 3 d}$ ) did not significantly differ from wildtype at P28 (Fig. 2A). However, at older ages heterozygotes displayed a small, but significant elevation in the high frequencies compared to wildtype mice (Fig. 2A). C57BL/6 mice carry the Cdh23 $3^{\text {ahl }}$ allele which causes an age-related hearing loss, as can be seen in the wildtype mice at older ages (Fig. 2A). At older ages, such as P210 it is difficult to discriminate between the effects of $C d h 23^{a h l}$ and those of the Caprin $1^{\text {tm } 3 d / t m 3 d}$ on the auditory system. We therefore primarily focused on the age P28 in which to investigate the mechanisms underlying the functional deficit in Caprin $1^{\text {tm3d/tm3d }}$ mice.

A more detailed analysis of ABR waveforms at $24 \mathrm{kHz}$ (Fig. 2B) the frequency at which Caprin $1^{\text {tm } 3 \mathrm{~d} / \mathrm{tm} 3 \mathrm{~d}}$ mice exhibited a large significant elevation in hearing thresholds at P28 (27 \pm 4 vs $70 \pm 16 \mathrm{~dB}$ SPL, mean \pm SD, Caprin $^{+/+}$vs Caprin $1^{\text {tm3d/tm3d }}$ respectively), showed that the amplitudes of ABR waves I and III-V were reduced in Caprin $1^{t m 3 d / t m 3 d}$ mice. We quantified this reduced amplitude focusing on ABR wave I which in humans and most mammals is generally agreed to reflect the action potentials in auditory nerve fibres driven by activity at the IHC-SGN synapse ${ }^{25-27}$. In Caprin $1^{t m 3 d / t m 3 d}$ mice, ABR wave I amplitudes were greatly reduced compared to wildtype mice and this reduction became more pronounced as a function of increasing sound level (Fig. 2C). In parallel, a small increase in the latency of ABR wave I was observed in Caprin $1^{\text {tm3d/tm } 3 d}$ mice compared to wildtype (Fig. 2D). 
A

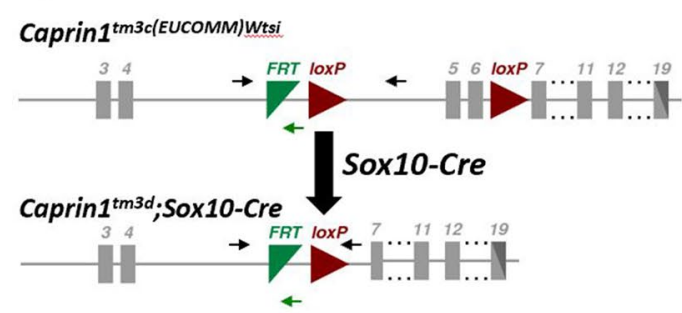

B

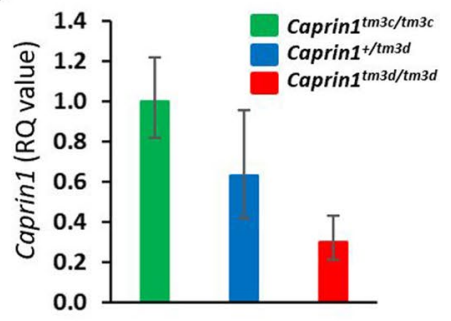

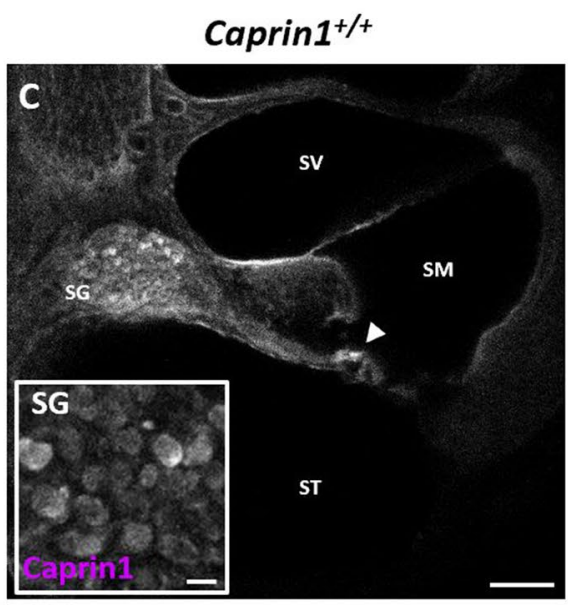
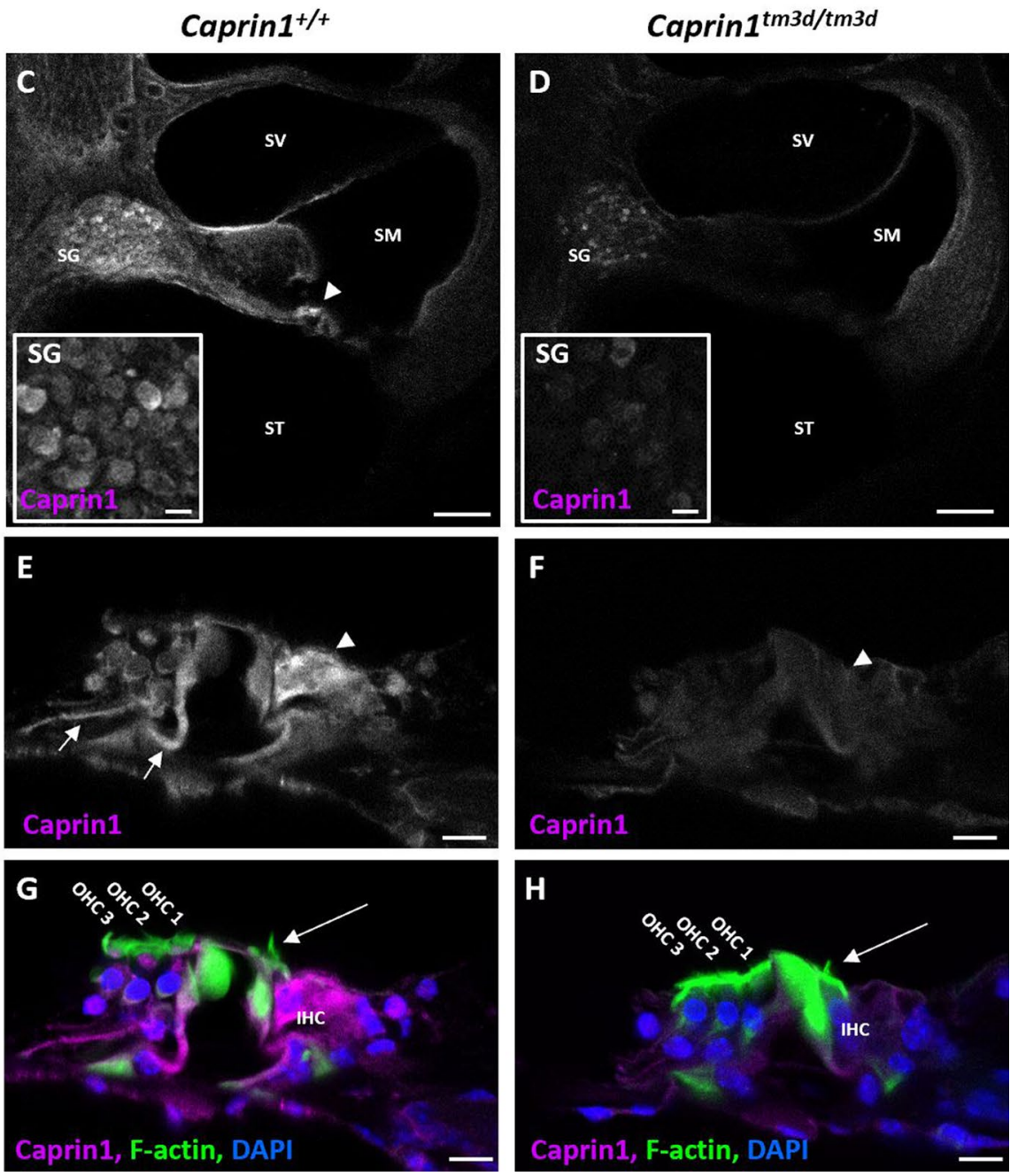

Figure 1. Targeted deletion of Caprin1 in the inner ear. (A) Schematic shows the design of the conditional allele for Caprin $1^{\text {tm } 3(\text { (EUCOMM)Wtsi }}$. Exons are shown in grey. LoxP sites flank the critical exons (exons 5-6) of the Caprin1 gene. The position of primers Caprin1_173389_F, Caprin1_173389_F and CAS_R1_Term are indicated by black and green arrows respectively. Mice carrying the conditional allele for Caprin 1 were crossed with mice expressing Cre-recombinase driven by the Sox 10 promoter deleting the floxed critical exons and generating a frameshift mutation in the inner ear (Caprin $1^{\text {tm } 3 d}$ ).

(B) Quantitative PCR of Caprin1 expression in the brain confirmed knockdown of Caprin1 in Caprin ${ }^{\text {tm3d/tm3d }}$ mice compared to control mice ( $\mathrm{p}=2 \mathrm{E}-06$, T-test comparing $\Delta \mathrm{CT}$ values). Heterozygotes displayed Caprin 1 levels intermediate to Caprin $1^{\text {tm } 3 d / t m 3 d}$ mice and Caprin ${ }^{\text {tm } 3 c / t m 3 c}$ controls. $R Q$, relative quantification adjusted to endogenous control values, error bars show 95\% confidence intervals. (C-H) Immunofluorescence labelling with anti-Caprin1 on vibratome sections of inner ears from P28 wild-type (C,E,G) and mutant (D,F,H) mice: anti-Caprin1 (magenta; for clarity shown alone in greyscale in C-F); DAPI (blue) and phalloidin staining of F-actin (green). Images are 3-focal plane averages from confocal Z-stacks. (C,D) Low magnification view of the basal cochlear coil, scale bar: $100 \mu \mathrm{m}$. $S V$ scala vestibuli, SM scala media, $S T$ scala tympani, $S G$ spiral ganglion. In the wild-type cochlea Caprin1 immunoreactivity is predominantly localized to the inner hair cells (arrowhead) and SG. Insets show higher magnification images of Caprin1 labelling in the SG. Note that a small number of spiral ganglion neurons continue to express Caprin1 in the mutant cochlea (inset in D). Scale bar for insets, $10 \mu \mathrm{m}$. (E-H) High magnification of the organ of Corti from the mid-cochlear coil, scale bar $10 \mu \mathrm{m}$. (G,H) show the three-label merge from (E,F) respectively. (E) Caprin1 immunoreactivity in the wild-type organ of Corti is detected particularly in IHCs (arrowheads, with clear actinrich stereocilia see in $\mathrm{G}$ and $\mathrm{H}$, long arrows) and also appears concentrated in the Deiter's cell region (arrows). (F) Minimal Caprin1 immunoreactivity was detected in the mutant organ of Corti. 


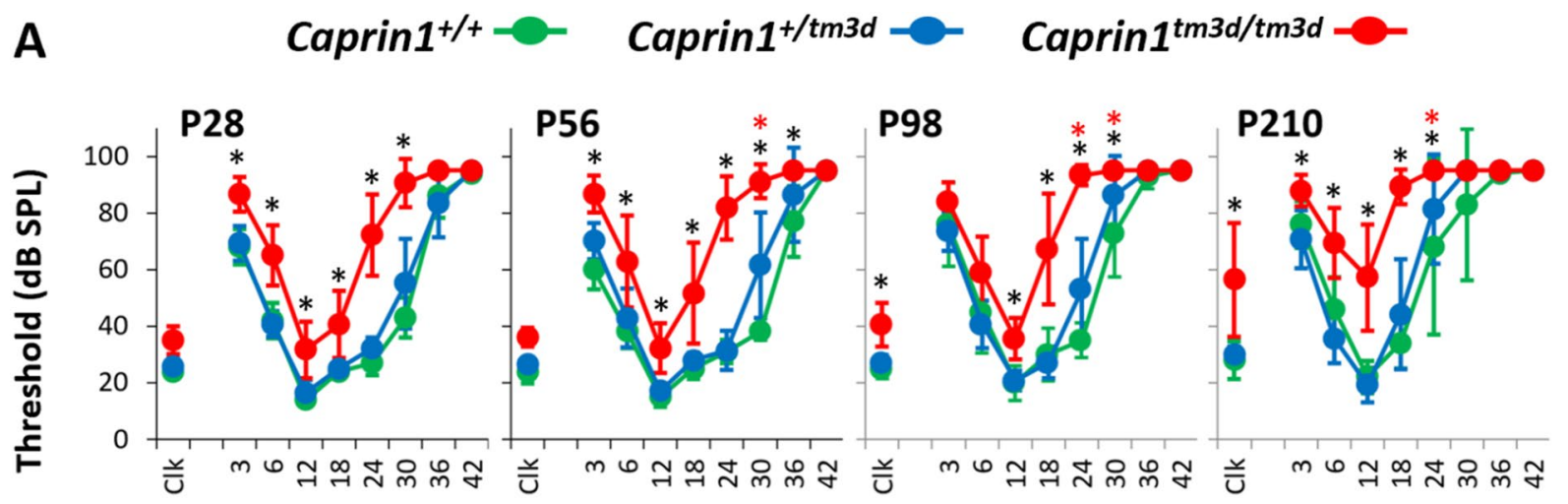

Stimulus $(\mathrm{kHz})$

B

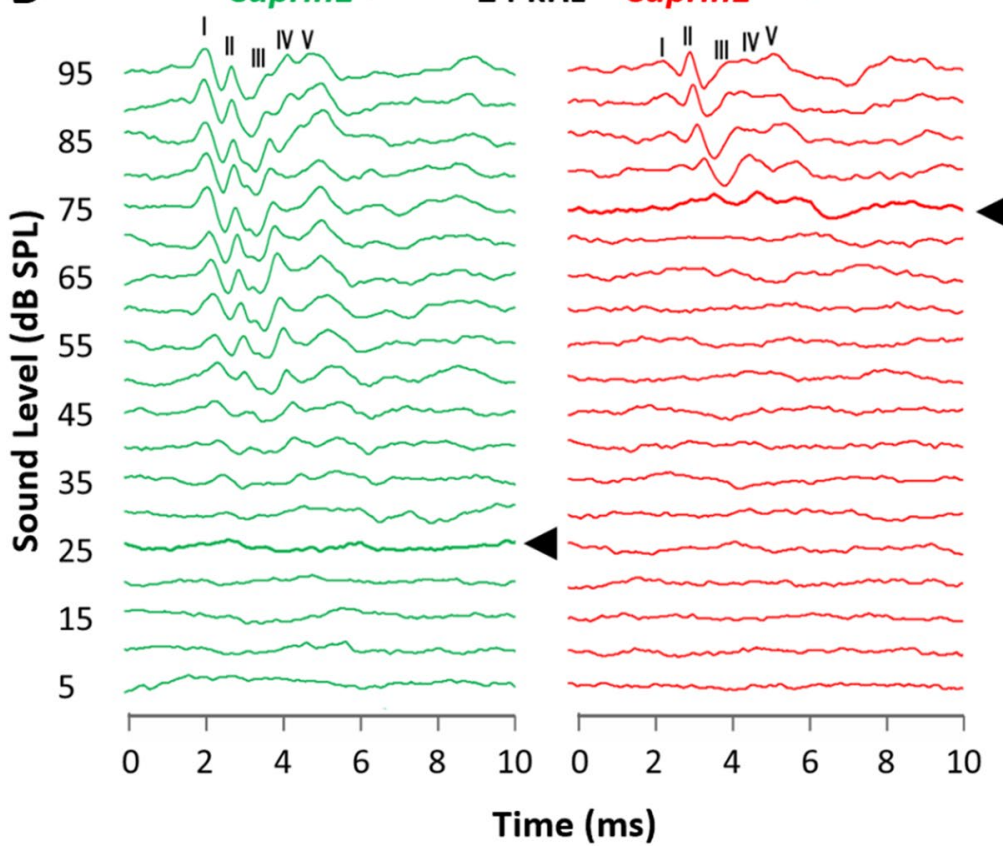

C

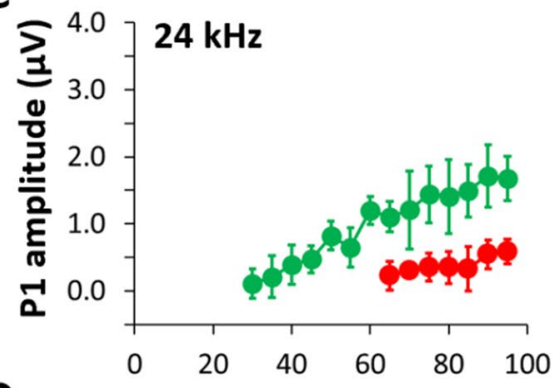

D

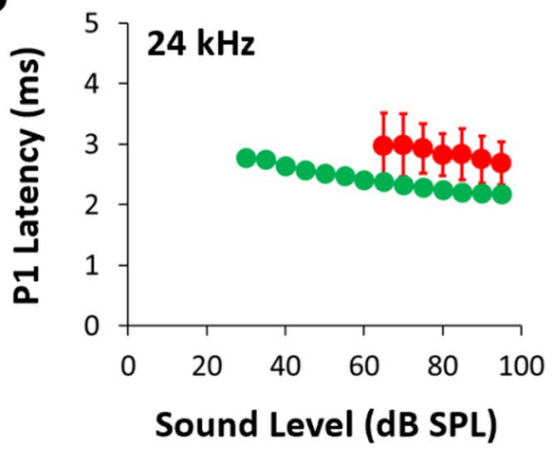

Figure 2. Caprin1-deficient mice exhibit a progressive hearing loss. (A) Mean ABR thresholds $( \pm S D)$ for click and pure-tone stimuli for Caprin $1^{+/+}(\mathrm{n}=5)$, Caprin1 $1^{+/ t m 3 d}(\mathrm{n}=7)$ and Caprin $1^{\text {tm3d/tm3d }}(\mathrm{n}=9)$ mice at P28, P56, P98 and P210 (Caprin 1 ${ }^{\text {tm3d/tm3d }}, \mathrm{n}=7$ at P210; 2 mice died before P210). ${ }^{*}$ mean hearing thresholds that significantly differ between Caprin $1^{+/+}$and Caprin $1^{\text {tm } 3 d / t m 3 d}$ mice; $*$ mean hearing thresholds that significantly differ between Caprin $1^{+/+}$and Caprin $1^{+/ t m 3 d}$ mice $(\mathrm{p}<0.05)$. (B) Representative ABR waveform traces obtained from Caprin $^{+/+}$and Caprin $1^{\text {tm } 3 d / t m 3 d}$ mice at P28 in response to a $24 \mathrm{kHz}$ pure tone presented from 0 to $95 \mathrm{~dB}$ SPL in $5 \mathrm{~dB}$ increments. The five positive peaks of the ABR waveform trace are numbered I to V. The ABR threshold is denoted by the thick line and arrowhead: $25 \mathrm{~dB}$ SPL versus $75 \mathrm{~dB}$ SPL in Caprin $1^{+/+}$and Caprin $1^{\text {tm } 3 d / t m 3 d}$ mice, respectively. (C) The growth of ABR wave I amplitude (P1 amplitude) and (D) the change in ABR wave I (P1) latency as a function of the sound pressure level $\left(\mathrm{dB}\right.$ SPL) in Caprin $1^{+/+}(\mathrm{n}=5)$ and Caprin1 ${ }^{\text {tm } 3 d / t m 3 d}(\mathrm{n}=8$, response completely absent in one mouse) mice at P28 in response to $24 \mathrm{kHz}$ stimuli is shown. $(\mathbf{C})$ Amplitudes $(\mu \mathrm{V})$ and $(\mathbf{D})$ latencies $(\mathrm{ms})$ are plotted as mean values; error bars: $\pm 1 \mathrm{SD}$.

Caprin1-deficient mice exhibit a post-synaptic defect. To characterise the functional deficit that underlies the hearing loss in Caprin1-deficient mice we used both cellular and sub-cellular markers in whole mount preparations of the organ of Corti. The cochlea is organized in a tonotopic gradient with low frequencies detected towards the apex and higher frequencies towards the base. We focused our investigations on the mid-basal cochlear coil at P28, which includes the $24 \mathrm{kHz}$ region, a region which exhibited a severe functional deficit at this time-point (Fig. 2A). First, we examined the general architecture of the organ of Corti. Phalloidin staining of filamentous actin (F-actin) revealed that the epithelial surface appeared normal with no obvious abnormality in the gross morphology of the organ of Corti in Caprin $1^{\text {tm3d/tm3d }}$ mice compared to wildtype mice. 

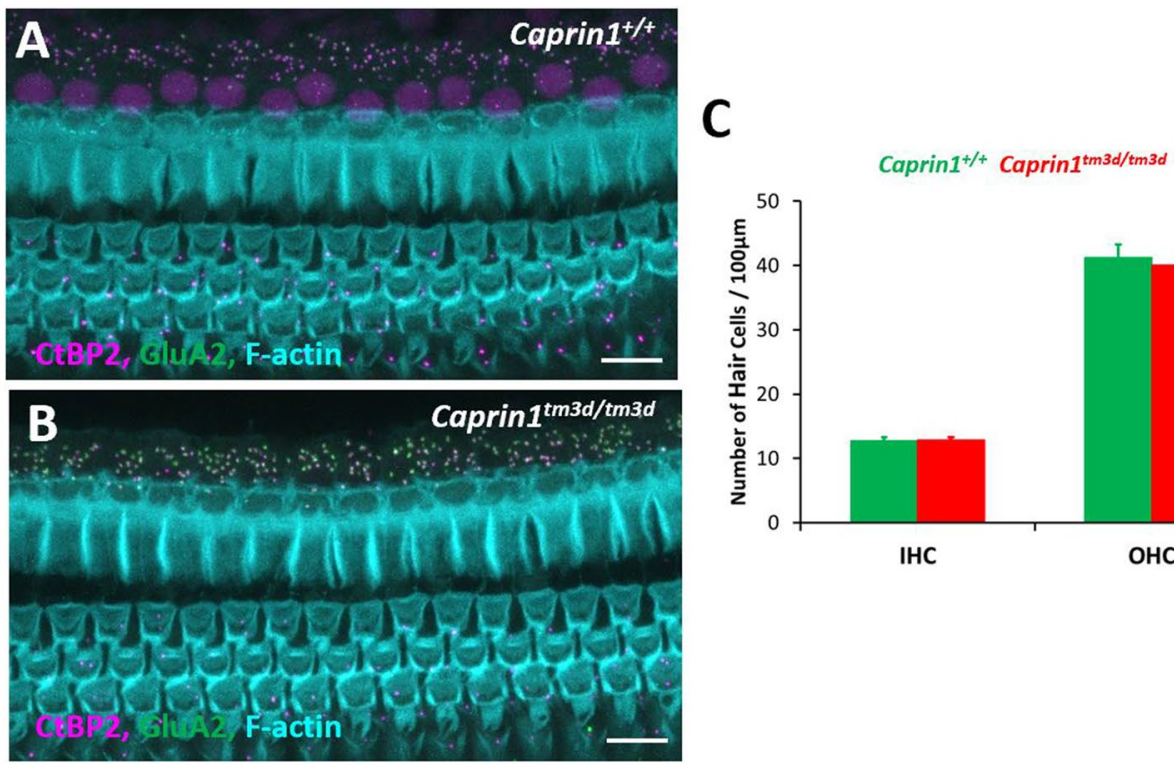

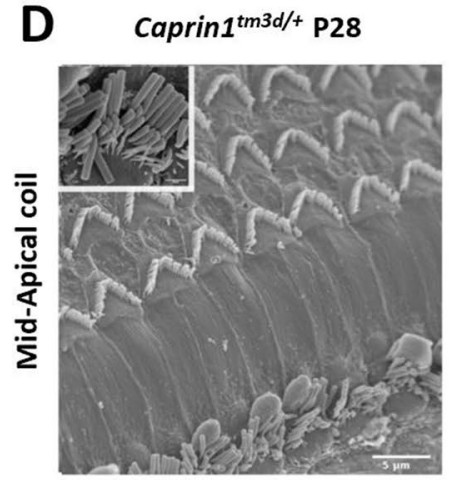

Caprin1 ${ }^{\text {tm3d/tm3d }}$ P28
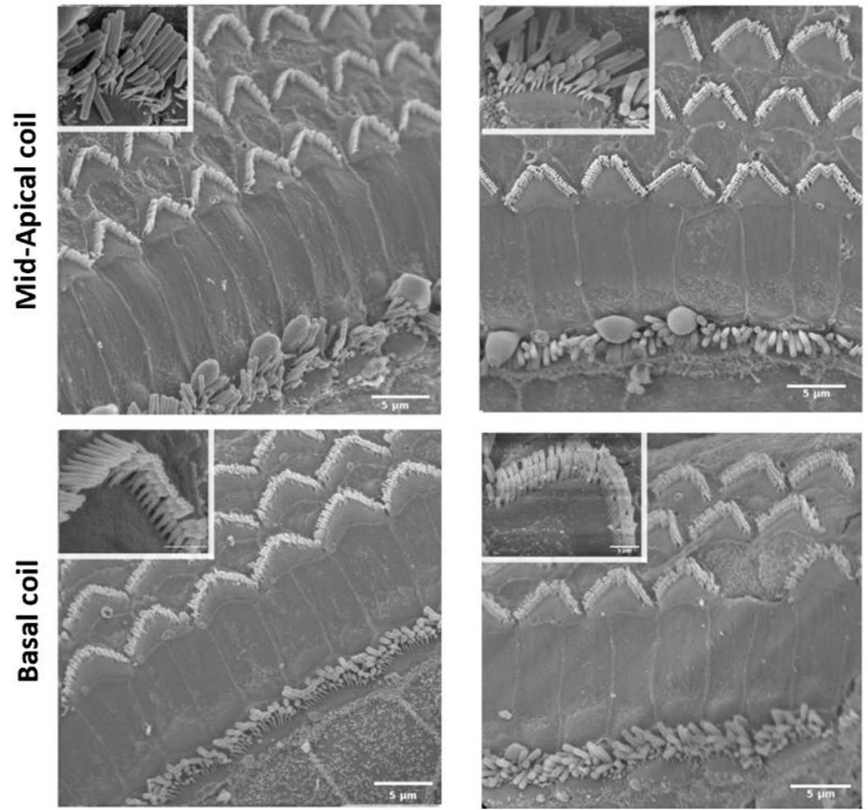

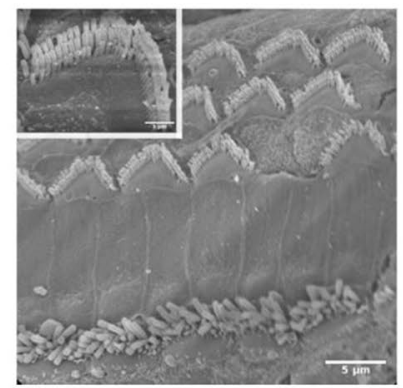

Caprin1 $^{\text {tm3d/tm3d }}$ P98
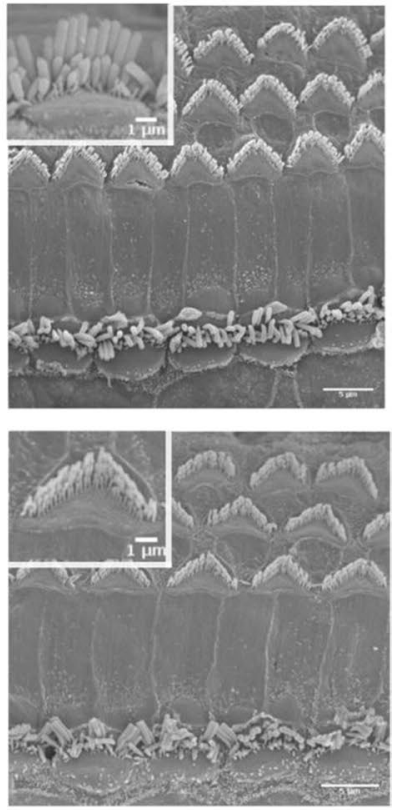

Figure 3. Caprin1-deficient mice exhibit normal hair cell morphology (A,B) Maximum projection images of immunofluorescence confocal Z-stacks from the mid-basal cochlear coil ( $24 \mathrm{kHz}$ region at P28) reveal the normal F-actin pattern (F-actin rich hair bundles, cuticular plates and pillar cell surfaces) of the reticular lamina in Caprin $1^{+/+}$and Caprin $1^{\text {tm3d/tm3d }}$ mice. Note subset regions from $(\mathbf{A}, \mathbf{B})$ are further described in Fig. $4 \mathrm{~A}-\mathrm{H}$. The number of IHCs and OHCs per $100 \mu \mathrm{m}$ length of the organ of Corti was not significantly different between Caprin $^{+/+}$and Caprin $1^{\text {tm3d/tm3d }}$ mice (C). SEM micrographs of the surface of the organ of Corti (D) reveal normal morphology in Caprin $1^{t m 3 d / t m 3 d}$ mice i.e. with v-shaped stereociliary hair bundles in OHCs and the 'balustrade'-like hair bundles of IHC in both basal and mid-apical cochlear turns at P28 (Caprin ${ }^{+/ t m 3 d}$ mice are used for comparison in these experiments) and also in older mice (P98). Scale bar: (A,B) $10 \mu \mathrm{m}$ (D).

Organisation of both IHC and outer hair cell (OHC) rows appeared normal (Fig. 3A,B) and labelling with an antibody to Myosin7a revealed normal appearance of IHC cell bodies (Fig. 4C,D). Quantification of IHCs and OHCs revealed no difference in the number of sensory hair cells between Caprin $1^{\text {tm } 3 d / t m 3 d}$ and wild-type mice (Fig. 3C). Similarly, scanning electron microscopy showed normal appearance of stereociliary hair bundles in Caprin $1^{\text {tm } 3 d / t m 3 d}$ mice at P28 either in basal or more apical regions (Fig. 3D). The stereociliary bundles of hair cells also appear to be normal without any loss of hair cells in older (P98) Caprin $1^{\text {tm } 3 d / t m 3 d}$ mice (Fig. 3D). We then examined the IHC synapse using antibodies to CtBP2 to label pre-synaptic IHC ribbons ${ }^{28}$, and to GluA2 (Fig. 4) 


\section{Caprin1 ${ }^{+/+}$}
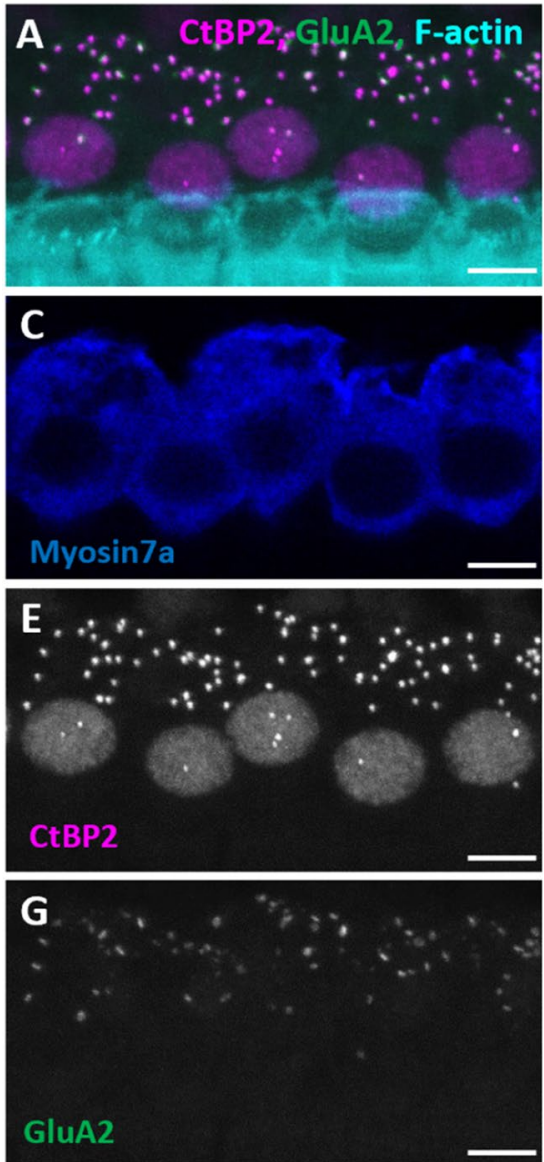

L

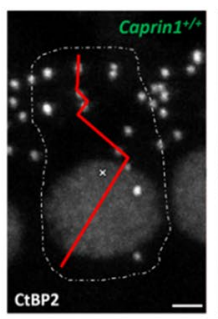

M

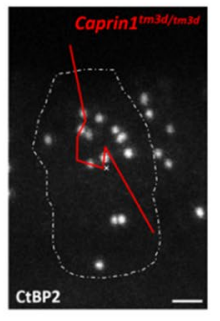

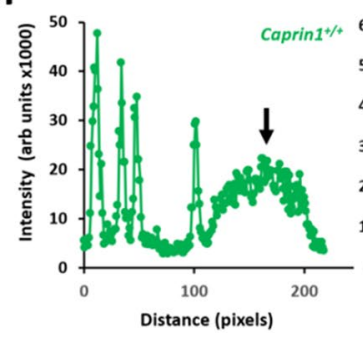

\section{Caprin $1^{\text {tm3d/tm3d }}$}
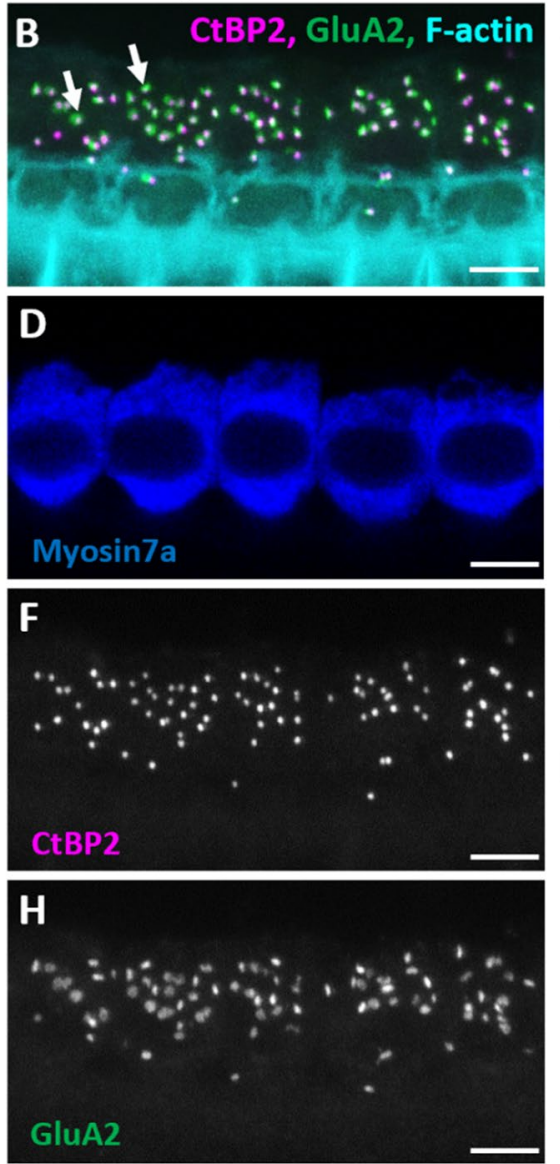

GluA2

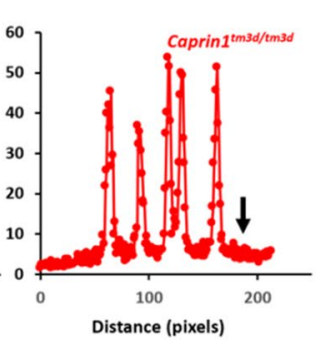

Caprin1 $1^{+1+}$

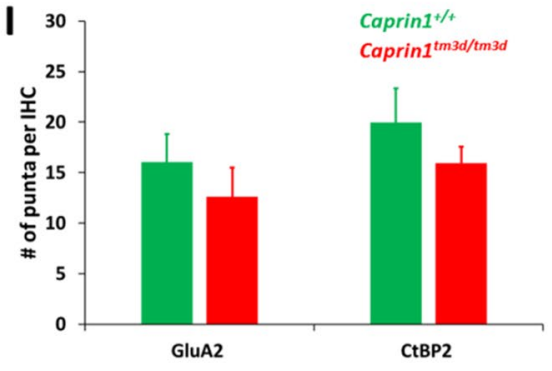

J 0.

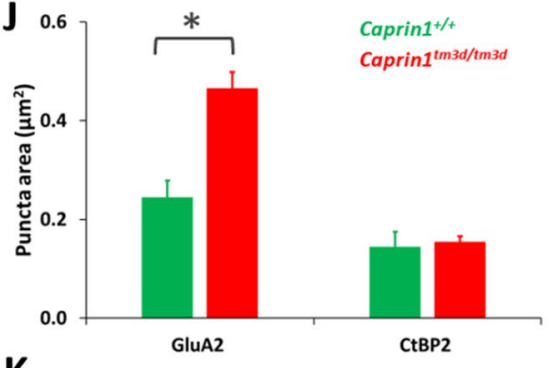

K

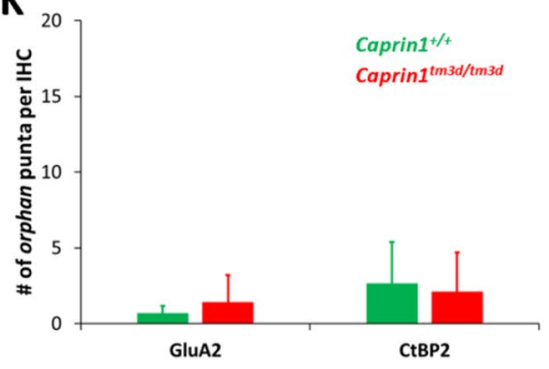

N

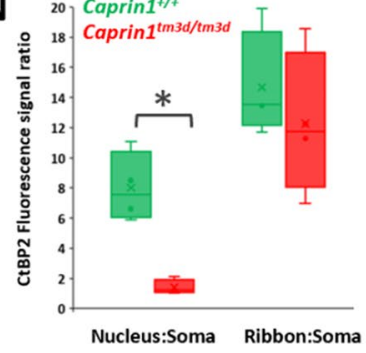

Figure 4. Caprin1-deficient mice exhibit a post-synaptic defect. Maximum projection images of immunofluorescence confocal Z-stacks from the mid-basal cochlear coil ( $24 \mathrm{kHz}$ region) from Caprin ${ }^{+/+}$ $(\mathbf{A}, \mathbf{C}, \mathbf{E}, \mathbf{G})$ and Caprin1 $1^{\text {tm } 3 d / t m 3 d}(\mathbf{B}, \mathbf{D}, \mathbf{F}, \mathbf{H})$ mice. Labelling with anti-CtBP2 and anti-GluA2 at P28 reveals alterations in the inner hair cell synapses with significantly enlarged GluA2 puncta (indicated by arrows in $(\mathbf{B}, \mathbf{G}-\mathbf{H})$ in Caprin $1^{\text {tm3d/tm3d }}$. There are no obvious abnormalities in the IHCs, labelled with anti-Myosin7a (C,D). Quantification of images from both Caprin $1^{+/+}(n=4)$ and Caprin $1^{\text {tm3d/tm3d }}(\mathrm{n}=5)$ mice reveals no significant difference between the number of pre-synaptic CtBP2-positive ribbons or the number of post-synaptic GluA2 receptor puncta (I) per IHC. The cross-sectional area of IHC pre-synaptic ribbons was not affected, however there was a significant difference in the size of post-synaptic GluA2 receptors between Caprin $1^{+/+}$and Caprin $1^{\text {tm } 3 d / t m 3 d}$ mice $(\mathbf{J}),{ }^{*} \mathrm{p}=0.0001$. Quantification of the numbers of orphan (unpaired) puncta revealed no significant differences between Caprin1 ${ }^{+/+}$and Caprin $1^{\text {tm3d/tm3d }}$ mice (K). The CtBP2-immunofluorescence intensity across the IHC soma in Caprin $1^{+/+}$mice showed a typical intensity profile (L,M, left panels) from minimal expression in the cytoplasm (soma) increasing to $\sim 8 \times$ greater signal in the nucleus and $\sim 14 \times$ greater signal in the ribbon puncta. Calculating the expression ratios between the nucleus:soma and the ribbons:soma (N) shows that CtBP2 expression was much reduced in Caprin $1^{\text {tm } 3 d / t m 3 d}$ IHCs (significant difference from Caprin $1^{+/ *} \mathrm{p}=0.009$ whereas the average intensity of the ribbon puncta was not significantly different from

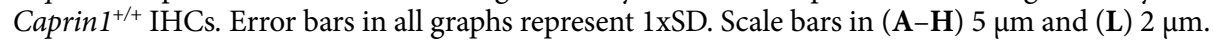


to label post-synaptic AMPA-type ionotropic glutamate receptors on adjacent afferent dendrites of type I spiral ganglion neurons (SGNs) ${ }^{29}$. The number of CtBP2-positive puncta and GluA2-positive puncta per IHC were reduced in Caprin $1^{\text {tm } 3 d / t m 3 d}$ mice compared to wildtype; this difference was not statistically significant but was at the border of statistical significance for CtBP2 (Fig. 4I, $\mathrm{p}=0.05$ for CtBP2 and $\mathrm{p}=0.12$ for GluA2). We then compared the mean cross-sectional area of the CtBP2- and GluA2-positive puncta and found that the GluA2-positive post-synaptic densities were almost twofold larger in Caprin $1^{\text {tm3d/tm3d }}$ mice compared to wildtypes (Fig. 4J: $0.47 \mathrm{SD} \pm 0.03 \mu \mathrm{m}^{2}$ versus $0.24 \mathrm{SD} \pm 0.03 \mu \mathrm{m}^{2}$, respectively; $\mathrm{p}=0.0001$ ). We did not observe a difference in size of the pre-synaptic CtBP2-positive puncta between Caprin $1^{\mathrm{tm} 3 d / t m 3 d}$ and wild-type mice (Fig. $\left.4 \mathrm{~J}\right)$. We also find no evidence to suggest a misalignment of pre and post synaptic components; there is no significant difference in the number of colocalised synapses between Caprin1 CKO mice and controls or in the number of orphan CtBP2 puncta or orphan GluA2 puncta (Fig. $4 \mathrm{~K}$ ). However, immunofluorescence images suggested that there was a consistent decrease in the expression of nuclear localized CtBP2 in IHC of Caprin $1^{\text {tm3d/tm3d }}$ compared to wildtype mice (Fig. 4E,F). Quantitative analysis of the relative intensity of the CtBP2 fluorescent signal in IHC nuclei and synaptic ribbons compared to the IHC cytoplasm in Caprin1 ${ }^{\text {tm3d/tm3d }}$ and wildtype mice (Fig. 4L) showed the CtBP2 fluorescent signal was significantly reduced in IHC nuclei of Caprin $1^{\text {tm } 3 d / t m 3 d}$ mice whereas the relative level of the expression in the synaptic ribbons was similar between wildtype and Caprin $1 \mathrm{cKO}$ mice (Fig. 4M,N).

Caprin1 deficient mice do not recover from noise-induced hearing loss. Given the progressive nature of the hearing loss in the Caprin $1^{\text {tm3d/tm3d }}$ mice and Caprin1's role in regulating translation during cellular stress, we tested whether these mice are more vulnerable to stress, in the form of acoustic trauma. We exposed Caprin ${ }^{t m 3 d / t m 3 d}$ mice to two different noise exposure paradigms (Fig. 5). The first is designed to induce a temporary threshold shift (TTS $)^{30,31}$ from which wildtype mice are known to recover (Fig. 5A,C). The second paradigm is designed to induce a permanent threshold shift (PTS) ${ }^{32,33}$ from which wildtype mice would not completely recover (Fig. 5B).

ABR thresholds in wildtype and Caprin $1^{t m 3 d / t m 3 d}$ mice were significantly elevated $24 \mathrm{~h}$ following the TTS paradigm, particularly at $24 \mathrm{kHz}$ (Fig. 5A). However, by 2 weeks post-exposure the elevated ABR thresholds in wildtype mice had recovered (Fig. 5A). In contrast, auditory sensitivity had not fully recovered in Caprin $1^{\text {tm } 3 d / t m 3 d}$ mice after the same 2-week period. The mean ABR thresholds at $24 \mathrm{kHz}$ remained significantly elevated at 2 weeks compared to pre-exposure measures; $63 \pm 7$ vs $48 \pm 34 \mathrm{~dB}$ SPL, respectively; $\mathrm{p}=0.0421$ (Fig. 5A). Subtraction to reveal the threshold shifts confirmed recovery of hearing thresholds in wildtype mice, but not Caprin $1^{\text {tm } 3 \mathrm{~d} / \mathrm{tm} 3 \mathrm{~d}}$ mice (Fig. 5C).

The TTS paradigm induces a TTS that predominantly localizes to the $24 \mathrm{kHz}$ region of the cochlea (Fig. 5D). To investigate the response of Caprin $1^{\text {tm } 3 d / t m 3 d}$ cochlea to greater stress we increased the extent of the acoustic trauma to a PTS paradigm (Fig. 5B). The PTS paradigm induces a substantial threshold shift in the mid-frequency region of the cochlea. ABR thresholds in wildtype and Caprin $1^{t m 3 d / t m 3 d}$ mice were elevated across $12-24 \mathrm{kHz}$ and in response to broadband click stimuli $24 \mathrm{~h}$ following exposure (Fig. 5B, see Clk). However, two weeks later ABR thresholds in wildtype mice were still elevated although they had recovered such that they did not significantly differ from pre-exposure levels (Fig. 5B). In comparison, the elevated ABR thresholds in Caprin $1^{\text {tm } 3 d / t m 3 d}$ mice show little evidence of recovery and remained significantly elevated, even appearing to worsen after 2 weeks (Fig. 5B, see Clk). These results suggest that the absence of Caprin1 protein in the cochlea impairs the ability of the cochlea to recover from acoustic stress.

To further understand why Caprin $1^{\text {tm3d/tm3d }}$ mice are unable to recover from noise exposure, we compared the effect of noise exposure on Caprin 1 protein expression and localisation in the wildtype and cKO cochlea. Two weeks following the TTS noise exposure paradigm pronounced Caprin 1 immunoreactivity was detected in both the IHC region (Fig. 6A,C, thick arrow) and the SGNs (Fig. 6E,G) in the wildtype cochlea. In noise-exposed cochleae, we observed clusters of Caprin 1 immunoreactivity localized to cytoplasm above the nucleus of the OHCs (thin arrow, Fig. 6A,C) and discrete punctate regions of Caprin 1 immunoreactivity were detected in the organ of Corti consistent with the appearance of stress granules (Fig. 6A,C short arrow). Negligible Caprin 1 immunoreactivity was observed in the organ of Corti (Fig. 6B,D) or SGN (Fig. 6F,H) of Caprin $1^{\text {tm } 3 d / t m 3 d}$ mice after noise exposure consistent with its inner ear cKO status. However, as described previously (Fig. 1C), a small fraction of SGN cells in noise-exposed Caprin $1^{t m 3 d / t m 3 d}$ mice continue to display Caprin1 immunoreactivity suggesting these cells escape Cre-targeted deletion (Fig. 6F,H, arrows).

Caprin1-deficient mice can still form stress granules. We have shown that Caprin $1^{\text {tm } 3 d / t m 3 d}$ mice fail to show normal recovery from two different acoustic stress paradigms when measured two weeks following noise exposure. This may be due to the significantly altered IHC-SGN post-synaptic morphology identified in Caprin $1^{t m 3 d / t m 3 d}$ mice but it could also be due to an effect of Caprin 1 knockdown on stress granule formation or function. The appearance of discrete aggregates of Caprin1-immunoreactivity in the organ of Corti of noiseexposed wild-type mice (Fig. 6) suggests that stress granule formation may play a role in auditory protection during noise exposure. Therefore, we tested the ability of the Caprin $1^{t m 3 d / t m 3 d}$ cochlea to form stress granules.

Previously we have shown that Caprin1-containing stress granules are induced in response to various types of cellular stress ${ }^{16,17}$. Aminoglycoside treatment triggers the formation of Caprin1-containing stress granules in ex-vivo postnatal rat cochlear explants ${ }^{17}$ and in-vivo in mouse hair cells ${ }^{16}$. Sodium arsenite, used to mimic oxidative stress, induces the formation of Caprin1-containing stress granules in UB/OC-2 cells (a mouse inner ear sensory epithelial cell line) and in ex-vivo cochlear explants ${ }^{16}$. Caprin1-containing stress granules colocalize with polyA + mRNA and known stress granule markers such as TIA-1 cytotoxic granule-associated RNA-binding protein (TIA-1) whereas in some cases Caprin1-positive granules do not contain TIA-1 ${ }^{16,17}$. Although Caprin 1 has been shown to be a key regulator of stress granule formation and its over-expression is sufficient to induce 
A

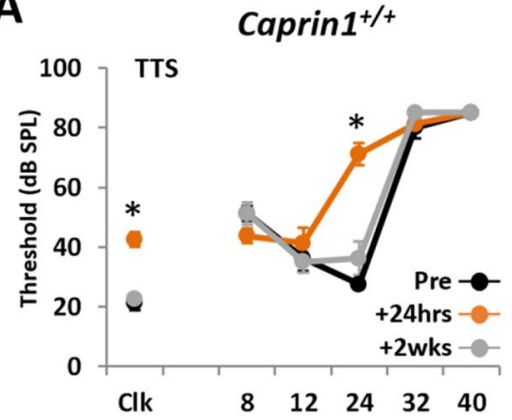

B

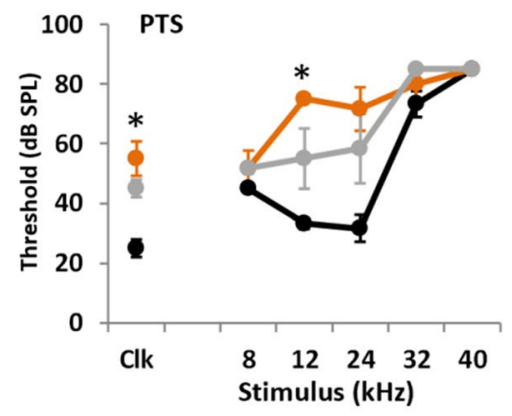

Caprin1 $^{\text {tm3d/tm3d }}$
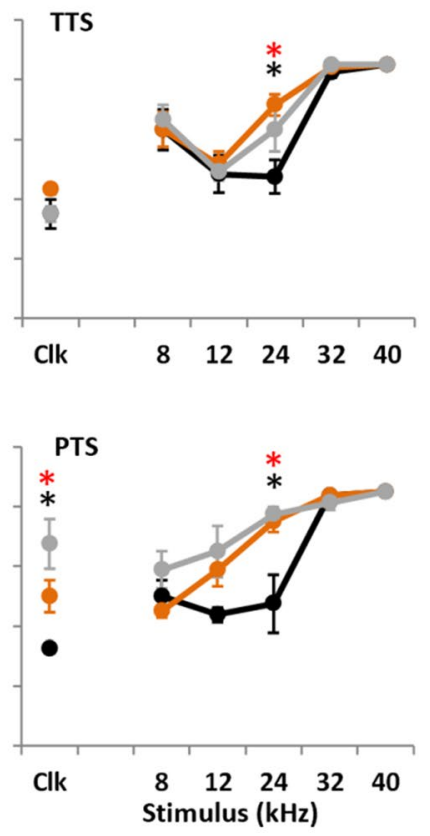

C

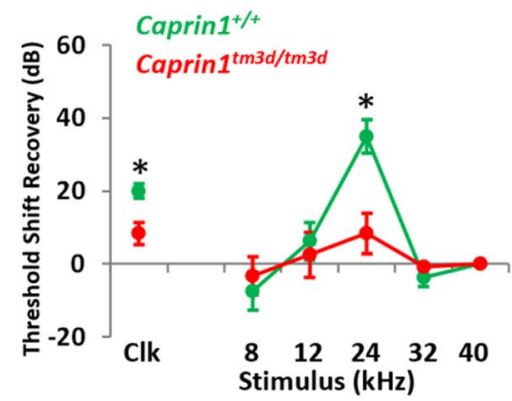

Figure 5. Caprin1 deficient mice do not recover from noise exposure. $(\mathbf{A}, \mathbf{B}) \mathrm{ABR}$ thresholds for broadband click (Clk) and tone pip stimuli $(8-40 \mathrm{kHz})$ for Caprin1 $1^{+/+}\left(\mathrm{n}=4\right.$ in $\mathbf{A}, \mathrm{n}=3$ in B), and Caprin $1^{\text {tm } 3 \mathrm{~d} / \mathrm{tm} 3 d}(\mathrm{n}=6$ in A, $n=4$ in B) mice following a temporary threshold shift (TTS, 8-16 kHz octave-band noise, $100 \mathrm{~dB} \mathrm{SPL}, 2 \mathrm{~h}$ ) in (A), or permanent threshold shift (PTS, 8-16 kHz octave-band noise, $110 \mathrm{~dB} \mathrm{SPL}, 3 \mathrm{~h}$ ) noise exposure regime in (B). ABRs were measured pre-noise exposure and at $24 \mathrm{~h}$ and 2 wks following noise exposure. ${ }^{*}$ and $*$ denote thresholds that are significantly elevated $(\mathrm{p}<0.05)$ at $24 \mathrm{~h}$ and 2 weeks post-noise exposure, respectively. $(\mathbf{C})$ Recovery of ABR threshold shifts for Caprin $1^{+/+}$and Caprin $1^{\text {tm } 3 d / t m 3 d}$ mice following TTS regime (calculated by subtracting the threshold at $24 \mathrm{~h}$ from that at $2 \mathrm{wks}) .{ }^{*}$ denotes threshold shifts that significantly differ $(\mathrm{p}<0.05)$ between Caprin $1^{+/+}$and Caprin $1^{\text {tm3d/tm3d }}$ mice. All data are mean \pm SEM.

stress granule formation, it is unclear whether it is an absolute requirement ${ }^{4,17}$. Hence, we investigated whether stress granules could still form in Caprin $1^{t m 3 d / t m 3 d}$-cochlea in response to cellular stress using two markers of stress granules, TIA- 1 and Human antigen $\mathrm{R}(\mathrm{HuR})^{9,34}$.

Sodium arsenite was applied to ex-vivo postnatal mouse cochlear explants to compare stress granule formation in Caprin $1^{\text {tm } 3 d / t m 3 d}$ and control (Caprin ${ }^{t m 3 c / t m 3 c}$, sox10-ve) mice (Fig. 7). We used this ex-vivo model to compare stress granule formation due to its more robust and consistent stress granule induction over in-vivo experiments. Cochlear explants from control mice exhibited robust stress granule formation in response to $1 \mathrm{~h}$ of sodium arsenite (Fig. 7). Abundant TIA-1-positive and Caprin1-positive (Fig. 7A) stress granules were observed in the $\mathrm{OHC}$ region and somewhat less robustly in the IHC region of control mice. HuR-positive stress granules followed a similar distribution pattern but appeared less widespread (Fig. 7B) than the Caprin-positive stress granules. Many TIA-1 and Caprin1-positive stress granules were observed (Fig. 7A, arrows) and a similar pattern was observed for Caprin 1 and HuR-positive granules (Fig. 7B, arrows). A similar distribution of TIA1-positive and HuR-positive stress granules was observed in Caprin ${ }^{\text {tm3d/tm3d }}$ cochleae compared to that of control mice (Fig. 7A,B). As expected, in Caprin ${ }^{t m 3 d / t m 3 d}$ cochleae the TIA-1- (Fig. 7A, arrowheads) or HuR- (Fig. 7B,C, white arrowheads) positive stress granules did not contain Caprin1, consistent with the conditional knockout phenotype of the Caprin ${ }^{\text {tm } 3 d / t m 3 d}$ mice. A small number of cells in the Caprin ${ }^{\text {tm3d/tm3d }}$ cochleae escaped the Cre 


\section{Caprin1 $1^{+/+}$}
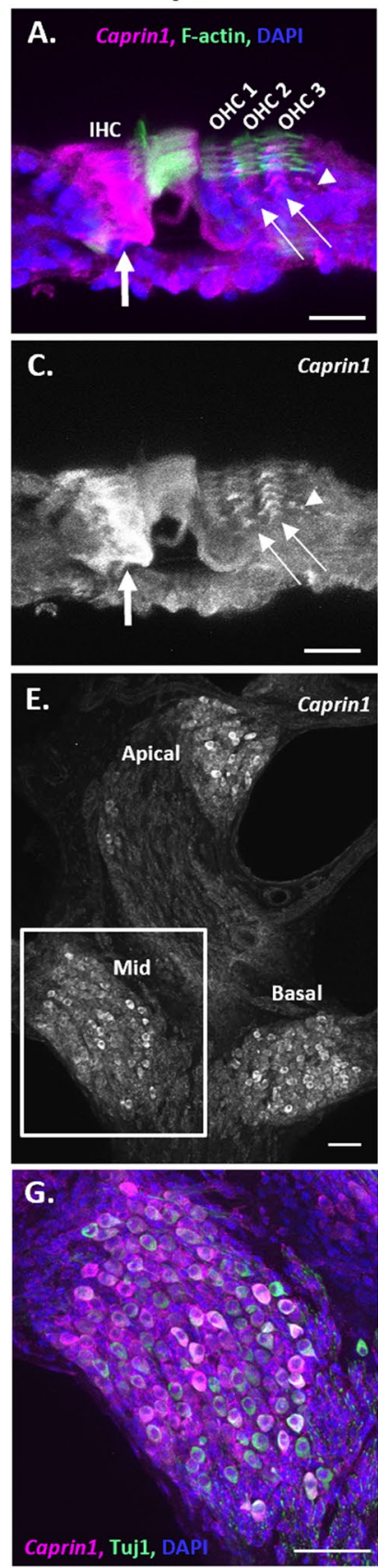

\section{Caprin $^{\text {tm3d/tm3d }}$}
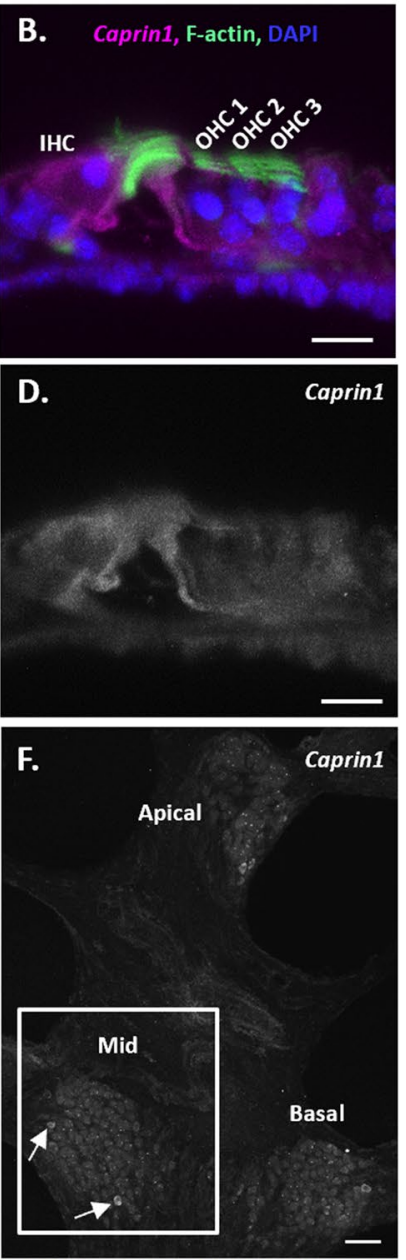

H.

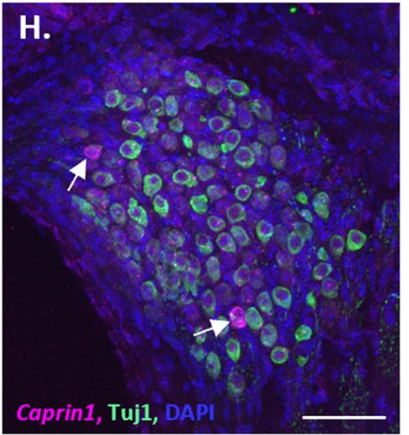

Figure 6. Expression of Caprin1 in the cochlea after noise exposure. (A-H) Results of immunofluorescence labelling with anti-Caprin1 on vibratome sections of wild-type $(\mathbf{A}, \mathbf{C}, \mathbf{E}, \mathbf{G})$ and $\mathrm{cKO}(\mathbf{B}, \mathbf{D}, \mathbf{F}, \mathbf{H})$ mouse organ of Corti (A-D) and SGN (E-H) at P44 two weeks following a TTS noise exposure regime. All images are representative confocal maximum projections. Anti-Caprin1 (white in $(\mathbf{C}-\mathbf{F})$; magenta in the merge $(\mathbf{A}, \mathbf{B}, \mathbf{G}, \mathbf{H})$; anti-Tuj1 labelling predominantly SGNs (green, G-H), DAPI (blue) and phalloidin labelling of f-actin (green, A,B). (A-D) The organ of Corti in the apical cochlear coil. (A,B) Show the merged image of $(\mathbf{C}, \mathbf{D})$ respectively. In the wild-type cochlea (A,C) abundant Caprin1 immunoreactivity is localized to the IHC region (thick arrow) and is also concentrated above the $\mathrm{OHC}$ nuclei (thin arrow, $\mathbf{A}, \mathbf{C}$ ). Evidence of punctate regions of Caprin 1 immunoreactivity were also detected (arrowhead, A,C). Minimal Caprin1 immunoreactivity was detected in the organ of Corti of Caprin $1^{\text {tm3d/tm3d }}$ mice $(\mathbf{B}, \mathbf{D})$. (E,F) a mid-modiolar vibratome section shows Caprin1 labelling of SGNs. The boxed regions are shown in $(\mathbf{G}, \mathbf{H})$, respectively. In the wild-type cochlea (E,G) Caprin1 immunoreactivity is localized to the cytoplasm of the majority of SGNs, across all cochlear coils, with some cells expressing more than others. In the Caprin $1^{\text {tm3d/tm3d }}$ cochlea, Caprin1 is not observed in SGNs $(\mathbf{F}, \mathbf{H})$, except for a few neurons that continue to exhibit Caprin 1 immunoreactivity (F,H, white arrows). IHC: inner hair cell; OHC: outer hair cell. Images represent data from at least 3 mice. Scale bars: $20 \mu \mathrm{m}$ in $(\mathbf{A}-\mathbf{F}), 50 \mu \mathrm{m}$ in $(\mathbf{G}, \mathbf{H})$. 
A
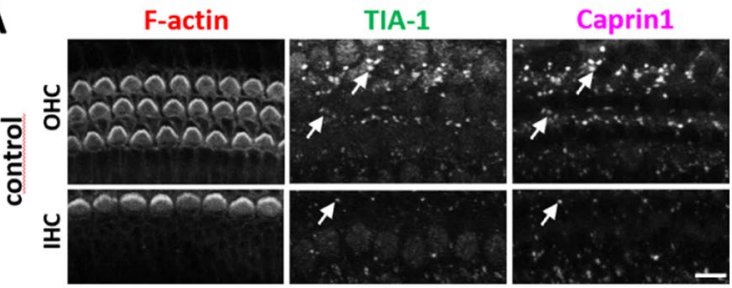

Orthogonal view - Merge
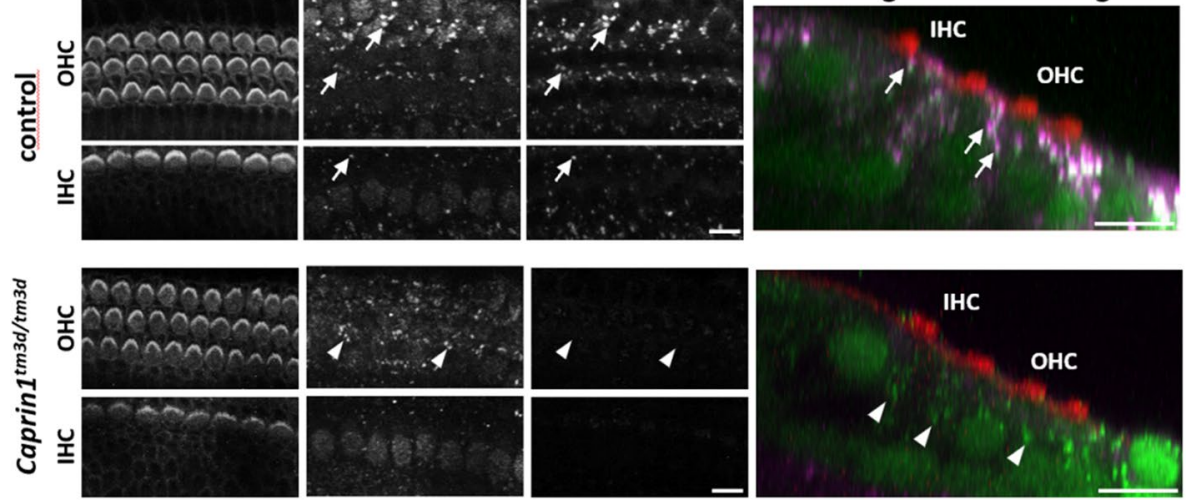

B

$$
\text { F-actin }
$$

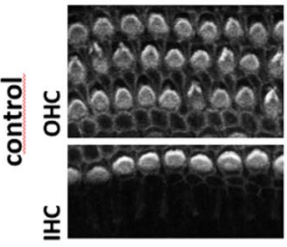

HuR

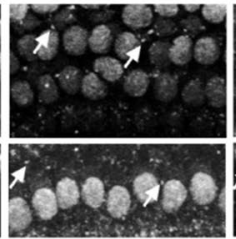

Caprin1
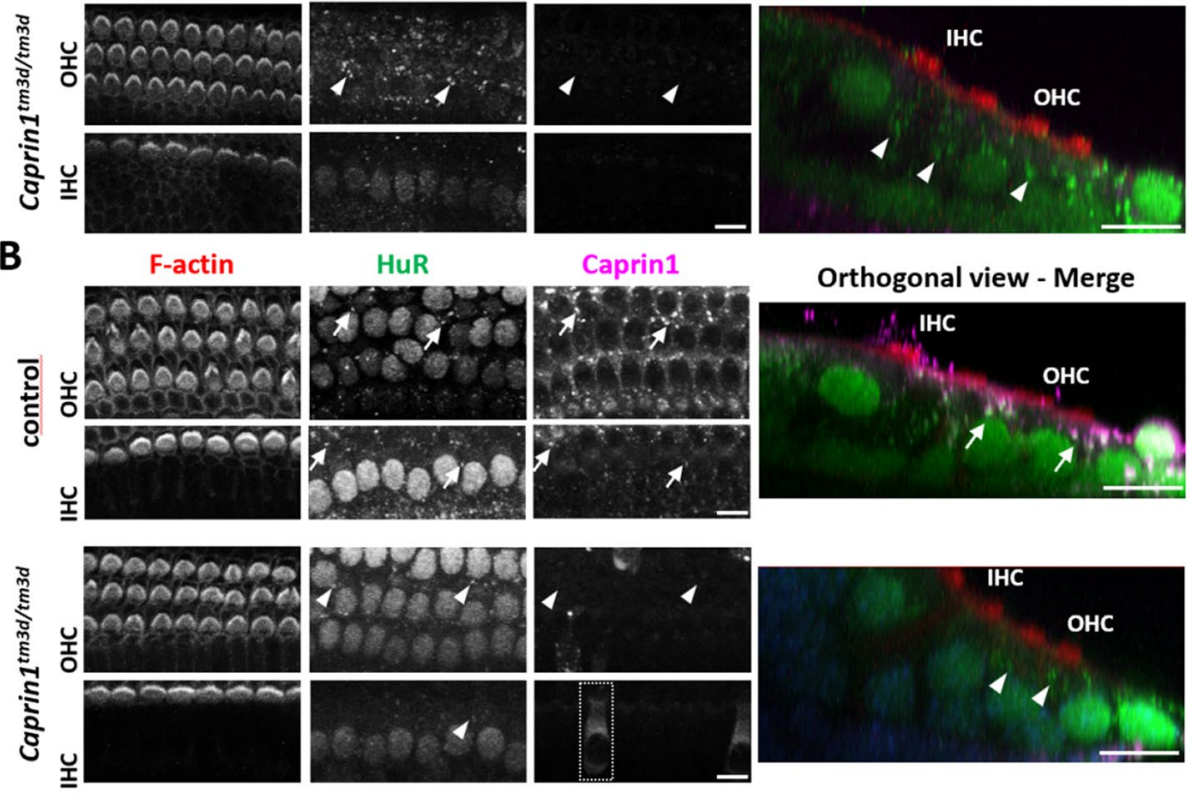

Orthogonal view - Merge

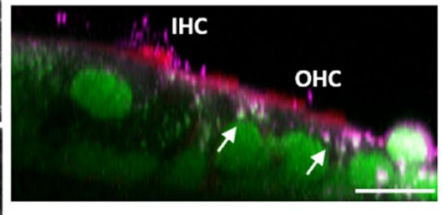

C
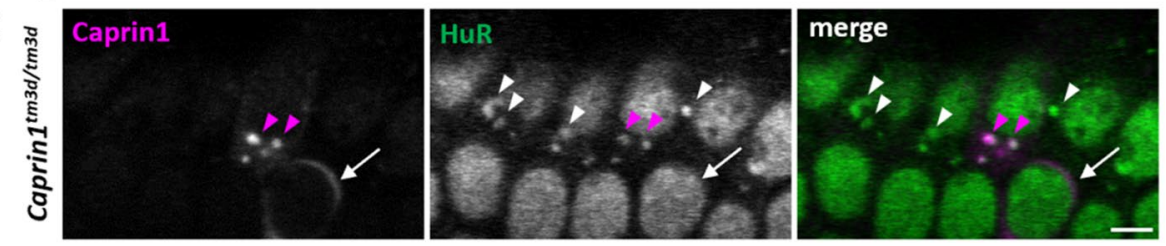

Figure 7. Caprin1-deficient mice can still form stress granules. Ex-vivo postnatal cochlear explants from control (see methods) and Caprin $1^{\text {tm } 3 d / t m 3 d}$ mice all treated with $0.5 \mathrm{mM}$ sodium arsenite for $1 \mathrm{~h}$ to induce stress granule formation. (A) Immunofluorescence maximum projections of confocal image stacks showing expression of Caprin 1 and TIA-1 (to label stress granules). Caprin $1^{\text {tm } 3 d / t m 3 d}$ mice show minimal expression of Caprin1 in the organ of Corti. In both control (arrows) and Caprin ${ }^{t m 3 d / t m 3 d}$ (arrowheads) explants TIA-1-positive stress granules form in the cells (including IHCs and OHCs) in response to arsenite treatment. In controls, Caprin1 and TIA-1 colocalise in the majority of stress granules. (B) Maximum projections of confocal image stacks showing expression of Caprin1 and an alternative stress granule marker HuR. The same pattern is observed with arsenite treatment, with HuR-positive/Caprin1-positive stress granules (arrows in controls) and HuR-positive stress granules (arrowheads) still formed in the Caprin $1^{\text {tm } 3 d / t m 3 d}$ hair cells (and other cell types). However, as in

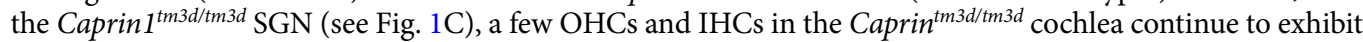
Caprin1-immunoreactivity consistent with Caprin1 protein expression again suggesting a few cells escape the Sox 10-Cre recombination (boxed region shows an example of an IHC). (C) Higher magnification view of Caprin $1^{\text {tm } 3 d / t m 3 d}$ cochlear cells showing identical HuR-positive stress granules (arrowheads) to the one "escaped" Caprin1-expressing cell neighbour cell (arrow). All explants (minimum $\mathrm{n}=3$ per experiment) were from the mid-basal cochlear coil and images were acquired from the mid-region of the explant. Scale bars, $(\mathbf{A}, \mathbf{B}) 10 \mu \mathrm{m}$.

recombination and expressed Caprin1. Stress granule formation in the $\mathrm{cKO}$ and Caprin1-expressing cells in the same explant was indistinguishable (Fig. 7C). These data suggest that stress granule formation in native cochlear cells is not dependent on Caprin 1.

\section{Discussion}

The stress granule RNA-binding protein Caprin1 is essential for maintenance of hearing. Stress granules are thought to play a protective role during cellular stress and dysregulation of their formation and disassembly has been implicated in a number of pathological processes, most notably neurodegeneration ${ }^{11,13,14}$. However, to date, our knowledge of stress granule biology is largely based on experiments performed in yeast or from manipulation of stress granule protein expression in various cell lines, using heat shock or sodium arsenite 
to induce stress granule formation. There is little corroboration of these findings from in-vivo work, especially in mammals in relation to native physiological stress. A limitation to these investigations has been that generation of knockout mutant mice for stress granule components such as Caprin1, G3BP1 and TIA-1 leads to embryonic or neonatal lethality ${ }^{5,35,36}$. Therefore, generation of the conditional knockout Caprin ${ }^{t m 3 d / t m 3 d}$ mouse model here represents one of the first opportunities to investigate the role of a stress granule protein in a mature mammalian system that is susceptible to physiological stress, the inner ear. Here, we report that conditional knockout of the stress granule RNA-binding protein Caprin1 in the inner ear leads to an early onset and progressive hearing loss in mice suggesting that it plays an important role in the protection and maintenance of the cochlea. The simplest explanation for the hearing loss we observed in Caprin $1^{\text {tm3d/tm3d }}$ mice is that the abnormal post-synaptic morphology we describe manifests itself as a defect in synaptic signaling between the IHC and the SGNs that is measured as a reduction in the ABR wave I and consequent overall hearing thresholds. We did not observe further evidence of changes in cellular architecture or function which might explain the auditory deficit (e.g. of the stria vascularis). However, as the Sox10-Cre will delete Caprin1 expression throughout the inner ear and craniofacial neural crest-derived cells ${ }^{23}$ we cannot exclude the possibility that there may be subtle alterations in the physiology of other cell types such as in the stria vascularis and glia that might also contribute to the phenotype.

In the absence of Caprin1, we find that cells within the cochlea can still generate stress granules in response to oxidative stress in vitro but we find that they are less able to recover from auditory stress in the form of noise exposure. To our knowledge this is the first report to demonstrate that conditional deletion of an RNA-binding protein and stress granule component leads to an increased susceptibility to extrinsic, cellular stress in vivo.

Under normal conditions, in the absence of an applied extrinsic stress, Caprin 1 is widely expressed in cochlear cells in the adult mouse inner ear consistent with the ubiquitous expression reported in other tissues ${ }^{3}$. However, some cells show a more intense staining suggesting increased Caprin 1 expression in the IHC region and SGNs (Fig. 1C). In the presence of extrinsic stress including noise and oxidative stress we found punctate staining of Caprin 1 in hair cells and in SGNs in response to noise in wildtype mice (Figs. 6 and 7). The punctate staining co-localizes with stress granule markers TIA-1 and HuR suggesting stress granule formation occurs in these cells in response to both types of stress. This is consistent with data showing that Caprin1-positive granules form in response to ototoxic aminoglycoside antibiotic exposure ${ }^{16,17}$. At the outset, we hypothesised that Caprin 1 and thus potentially stress granules, play an important homeostatic, protective role in maintenance of sensory receptor hair cells during different types of cellular stress. In support of this, in the absence of Caprin 1 within the inner ear, Caprin $1^{\text {tm } 3 d / t m 3 d}$ mice have a high-frequency hearing loss at 4 weeks that is progressive and extends into lower-frequencies with increasing age (Fig. 2A), a pattern of hearing loss which is commonly found in patients with age-related hearing loss. Heterozygous Caprin $1^{+/ t m 3 d}$ mice display similar hearing thresholds to Caprin $1^{+/+}$ mice except for a small elevation of thresholds at higher frequencies, although this does not progress markedly with age (Fig. 2A). Characterization of the cochlea of Caprin $1^{\text {tm } 3 d / t m 3 d}$ mice at P28 when they already have a significant hearing loss found no obvious loss of either IHC or OHC and the cellular architecture of the organ of Corti appeared normal. At P98, when Caprin $1^{\text {tm } 3 d / t m 3 d}$ mice have progressed to a profound hearing loss at $24 \mathrm{kHz}$ and above, the hair cells still appear well preserved in the corresponding mid-base region of the cochlea. These data indicate that the hearing loss does not result from a gross developmental defect and is more consistent with a deficit in auditory maintenance or protection.

Evidence that the IHC-SGN synapse is altered in Caprin1 $1^{\text {tm3d/tm3d }}$ mice. Reduced ABR wave I amplitudes in the presence of an otherwise normal audiogram is a characteristic feature of cochlear synaptopathy; a neuropathy whereby the synaptic connections between IHCs and the peripheral afferent dendrites of the SGNs are lost or damaged in response to noise exposure and/or the ageing process ${ }^{30,37-39}$. ABR wave I amplitudes were reduced in Caprin $1^{t m 3 d / t m 3 d}$ mice from P28, the earliest time-point assessed (Fig. 2) consistent with a cochlear synaptopathy. After detailed investigation in P28 mice using pre and post synaptic markers, we found that the GluA2 post-synaptic densities were abnormal, with GluA2-positive cross-sectional areas that were nearly twice as large in Caprin $1^{\text {tm3d/tm3d }}$ mice compared to wildtype $(\mathrm{p}<0.0001)$. We did not find a significant difference in the number of GluA2-labelled post-synaptic densities (at the terminals of the auditory nerve fibers that innervate IHCs) between Caprin $1^{\text {tm3d/tm3d }}$ mice and wildtype mice (Fig. 4). The values are consistent with the numbers reported in the literature for normal hearing mice; approximately 17 synapses per IHC for the midcochlear coil ${ }^{30}$. Additionally, there was no significant difference in the number of CtBP2-labelled pre-synaptic ribbons in IHCs, although we note that the reduction was at the borderline of significance $(\mathrm{p}=0.05)$ suggesting further investigation of the pre-synaptic ribbons is warranted, as this may be related to the GluA2 morphology difference. However, unlike the post-synaptic elements, the average size of the CtBP2-labelled pre-synaptic ribbons was unchanged in cKO mice compared to wildtype.

GluA2 is a crucial component of the AMPA-type ionotropic glutamate receptor, the major excitatory synaptic receptor in the auditory system, crucial for transmission of sound-induced activity from the cochlea to the auditory cortex ${ }^{40,41}$. As well as being a key regulator of stress granule formation Caprin 1 has been implicated in regulation of local RNA translation in neuronal RNA granules located at synaptic junctions $s^{5,7,42}$. In those studies, loss of Caprin 1 in hippocampal neurons led to loss of synaptic strength and a consequential deficit in long term memory formation in mutant mice. Using genome wide profiling of mRNA localization, Shiina, Yamaguchi et al. 2010, assigned the cause of this deficit to a lack of localization of dendritic mRNAs to synapses in the absence of Caprin1, resulting in changes in the distribution of synaptic proteins, particularly AMPA receptors, consistent with the changes we observe here. Combining those data with our own data from Caprin cKO mice described here suggests that Caprin 1 is responsible for regulating the local translation of critical synaptic proteins and hence maintaining the integrity and function of the primary auditory synapse. We suggest that when Caprin 1 expression is knocked down or absent a critical homeostatic control mechanism becomes dysregulated leading 
to changes in post-synaptic structure and function, the latter being consistent with the observed reduction in wave 1 amplitudes in cKO mice (Fig. 2C). In support of this homeostatic requirement, a unilateral reduction in sound-induced activity in the immature mouse cochlea (at p11) has previously been shown to modulate the size and the molecular make-up of the IHC-SGN synapse ${ }^{43}$. Similar to our observations in Caprin $1^{\text {tm } 3 d / t m 3 d}$ mice, the effect was restricted to the changes in the size of the post-synaptic densities labelled with GluA antibodies ${ }^{43}$.

A further striking and consistent difference that we observed in cKO mice was the loss of CtBP2 staining in the IHC nuclei of Caprin $1^{t m 3 d / t m 3 d}$ mice. The CtBP2 gene is subject to alternative splicing producing both short $(C t B P 2-S)$ and long $(C t B P 2-L)$ isoforms, in addition to a third isoform termed Ribeye which utilizes an alternative first exon ${ }^{44,45}$. Unlike the more widely expressed CtBP2 isoforms, Ribeye is primarily localized to ribbon synapses present in sensory cell-types and is critical to their function, including the IHC synapse ${ }^{44,45}$. In contrast, $C t B P 2-L$ contains a nuclear localization signal, and both $C t B P 2-S$ and $C t B P 2-L$ function as corepressors by interacting with transcription factors to repress their activity ${ }^{45}$. Currently, aside from Ribeye, little is known regarding the expression and function of the additional CtBP2 isoforms in the cochlea. The transcriptional repressor forms of CtBP2 promote cell survival via repression of pro-apoptotic genes Bax, Puma, Bik, and Noxa ${ }^{45}$. Our observations that Caprin ${ }^{t m 3 d / t m 3 d}$ mice exhibit a loss of nuclear CtBP2 expression suggests the presence of a nuclear isoform of the $C t B P 2$ gene in the cochlea that is modulated, directly or indirectly by Caprin1. How this loss of nuclear CtBP2 expression manifests itself at the level of the IHC and thus contributes to the auditory defect in Caprin $1^{\text {tm } 3 d / t m 3 d}$ mice needs further investigation but CtBP2's role in preventing apoptosis is one possibility. However, we did not observe a loss of IHCs associated with the hearing loss that we identified in Caprin $1^{\text {tm } 3 d / t m 3 d}$ mice (Fig. 3C). Furthermore, variation at the CTBP2 locus has also recently been identified in a genome wide association study of self-reported hearing loss in the UK Biobank Cohort suggesting it may play a role in auditory maintenance ${ }^{46}$. The most significantly associated variant lies within the first intron of $C t B P 2-L$, some distance upstream of the RIBEYE transcript which also raises the possibility that the transcriptional repressor isoforms may be required for normal cochlear function.

Stress granule formation in Caprin $1^{\text {tm3d/tm3d }}$ mice and its role in auditory protection. Environmental stress in the form of day-to-day sounds, and at the more extreme, noise exposure, is arguably the biggest extrinsic stressor to the inner ear and a major contributor to age-related hearing loss ${ }^{38,47}$. Intense noise exposure over hours damages hair cell stereocilia and leads to hair cell death and a permanent hearing loss ${ }^{33}$. However, moderate noise exposure typically results in a reversible hearing loss, with recovery of ABR thresholds but a persistent cochlear synaptopathy and reduced wave-I, which has been termed 'hidden hearing loss', since noise-exposed (or aged) humans may have normal pure-tone audiogram thresholds but have significant difficulties distinguishing speech sounds in background noise $e^{30,37,48}$. Here, we found that unlike wildtype mice, ABR thresholds in Caprin1 cKO mice fail to recover from such moderate noise exposure. Using that same noise paradigm, we observed prominent expression of Caprin 1 in the IHC region in wildtype mice, but not in the cKO. These data taken together suggest that Caprin 1 may be necessary for auditory protection during stress and/or for repair and recovery from stress-induced damage. Our data indicate that the failure of cKO mice to recover from noise exposure does not result from an inability to form stress granules since cKO cells were still able to do this (Fig. 7). Whether the absence of Caprin1 might alter the efficacy of the overall stress granule response remains to be determined but, since different RNA binding proteins are known to bind and recruit different populations of specific mRNAs to RNA granules and stress granules, it seems likely that the absence of Caprin 1 would alter the mRNA components of stress granules ${ }^{49,50}$. Our present data suggest that Caprin 1 plays a key role within stress granules or neuronal RNA granules and is a key determinant of the cochlea's homeostatic response to noise exposure. The data also suggest that the regulation of cochlear synaptic form and function is dependent on neuronal RNA granules and the function of Caprin1. Additionally, Caprin1 may regulate other molecular functions of IHCs, potentially through its effects on nuclear CtBP2. In future work it will be important to characterize the nature of the mRNA molecules recruited to cochlear RNA granules by Caprin1 during stress. In summary, the data suggest a key role for Caprin 1 acting pre- or post-synaptically to determine how the auditory system responds to and recovers from damage, revealing a potential therapeutic avenue for preventing acquired hearing loss.

\section{Materials and methods}

All experiments involving animals in this study were performed in accordance with the regulations of the U.K. Animals (Scientific Procedures) Act of 1986 (ASPA) approved by the King's College London Ethical Review Committee and the UCL Animal Welfare and Ethical Review Body and were carried out in compliance with the ARRIVE guidelines.

Generation and genotyping of Caprin 1 conditional knockout mice. Mice carrying a conditionalready allele for Caprin1 (Caprin $1^{\text {tm } 3 c(E U C O M M) W t s i}$; MGI ref: 5,692,641) on a C57BL/6 N; C57BL/6 N-A ${ }^{\text {tm } 1 B r d / a}$ background were generated at the Wellcome Sanger Institute by the Mouse Genetics Project ${ }^{51,52}$. Flp recombinasemediated excision of the cassette inserted into the Caprin $1^{\text {tm } 3 a}$ allele generated the Caprin $1^{\text {tm } 3 c}$ allele containing LoxP sites flanking exons 5-6 of Caprin1 (Fig. 1A; https://www.mousephenotype.org/data/genes/MGI:18582 34). Caprin ${ }^{t m 3 c}$ mice were crossed with Sox10-Cre mice ( $\mathrm{Tg}$ (Sox10-cre)1 Wdr; MGI ref: 3586900; from Prof. William Richardson, UCL). The Sox 10 gene is expressed in the otic vesicle around embryonic day $9.5^{23,53}$. Therefore, Cre-loxP recombination generates the Caprin $1^{\text {tm3d }}$ allele, deleting exons 5 and 6 in the inner ear ${ }^{24}$. The Sox10Cre mice used to breed with the Caprin $1^{t m 3 c}$ mice were maintained on a mixed genetic background including C57BL/6N and CBA. Aberrant homologous recombination occurs when Sox10-Cre is transmitted through the paternal germline ${ }^{54}$. Therefore, experimental $t m 3 d$ mice were generated by crossing female Caprin $1^{\text {tm } 3 d /+}$ 
or Caprin $1^{t m 3 d / t m 3 d}$ mice with Sox10-Cre negative males Caprin $1^{\text {tm } 3 c /+}$ or Caprin $1^{t m 3 c / t m 3 c}$. Experimental controls were either littermates wildtype for the Caprin $1^{\text {tm } 3 c}$ allele in the presence of the Sox10-Cre transgene (Caprin ${ }^{+/+}$ mice) or negative for the Sox10-Cre allele (Caprin $1^{\text {tm } 3 c / t m 3 c}$ mice). Both male and female animals were used in experiments. Mice were born in normal Mendelian ratios and no excessive deaths were recorded in any genotype group. Details of numbers of experimental animals used are given in legends.

PCR-based genotyping used DNA extracted from pinna tissue. PCRs were run in single-plex using primer pairs: Caprin1_173389_F5'-AGCCAGTGCTCTTTGAACCC-3' and Caprin1_173389_R 5'-GCCAAACATCCA CCACTGAC-3' generating a $600 \mathrm{bp}$ product at the native genomic locus and a $752 \mathrm{bp}$ product with the floxedallele for Caprin1; Caprin1_173389_F and CAS_R1_Term 5'-TCGTGGTATCGTTATGCGCC-3' generating a 236 bp product for the floxed-allele for Caprin1 (see Fig. 1); and Sox10Cre_F 5'-GCGGTCTGGCAGTAAAAA CTATC-3' and Sox10Cre_R 5'-GTGAAACAGCATTGCTGTCACTT-3' generating a 101 bp product in the presence of the Sox10-Cre transgene. Mice carrying the Caprin1 deletion (Caprin $1^{\text {tm } 3 d}$ allele) were confirmed by presence of the $236 \mathrm{bp}$ PCR band (Caprin $1^{\text {tm3c }}$ allele) in conjunction with the $101 \mathrm{bp}$ band (Sox10-Cre allele). PCR cycling conditions are available upon request.

Quantitative real-time PCR. Brain tissue from Caprin ${ }^{t m 3 d / t m 3 d}$, Caprin1 ${ }^{+/ t m 3 d}$ and Caprin1 $1^{\text {tm } 3 c / t m 3 c}$ (Sox10negative) mice ( $\mathrm{n}=3$ mice per genotype) was dissected in RNAlater (QIAGEN $\left.{ }^{\circ}\right)$, lysed and homogenized with a TissueRuptor and total RNA isolated using RNeasy ${ }^{\circ}$ Plus Mini Kit (QIAGEN $\left.{ }^{\circ}\right)$. cDNA synthesis was performed using the Omniscript ${ }^{\circ}$ RT Kit $\left(\right.$ QIAGEN $^{*}$ ) with random primers and rRNasin-Plus Ribonuclease Inhibitor (Promega). Caprin1 expression was determined by qPCR (Taqman gene expression assay Rn01512768-m1 (Applied Biosystems) using an SDS7500 Fast-PCR System (Applied Biosystems). Relative quantification of Caprin1 was based on triplicate samples using the $2^{-\Delta \Delta C t}$ method with eukaryotic $18 \mathrm{~S}$ rRNA as the endogenous control (Applied Biosystems, Cat. No: 4319413E).

Preparation of cochleae and immunofluorescence analysis. Auditory bullae were dissected and fixed in $4 \%$ paraformaldehyde (PFA) for $1 \mathrm{~h}$ at room temperature, washed in PBS and decalcified in $4.13 \%$ EDTA, pH7.4, in PBS for $72 \mathrm{~h}$ at $4{ }^{\circ} \mathrm{C}$. For cochlear whole-mounts, the organ of Corti was dissected into 4 halfcoils (apex; mid-apical; mid-basal and basal) using an adaptation of the Eaton-Peabody Laboratories protocol ${ }^{55}$. For vibratome sections, bullae were mounted in $4 \%$ low melting point agarose (Sigma-Aldrich, Gillingham, UK) and sectioned at $200 \mu \mathrm{m}$ (Intracel 1000-Plus Vibratome, Royston, UK). Vibratome slices were permeabilized and blocked with 0.5\% Triton-X 100, 10\% goat serum in PHEM buffer (60 mM PIPES, 25 mM Hepes, 10 mM EGTA, $2 \mathrm{mM} \mathrm{MgCl}_{2}, \mathrm{pH}$ 6.9) for $2 \mathrm{~h}$ at room temperature. Cochlear whole-mounts were permeabilised with $5 \%$ tween20 in PBS for $1 \mathrm{~h}$ at room temperature and blocked with 10\% horse serum, $0.5 \%$ Triton-X 100 in PBS for $2 \mathrm{~h}$ at room temperature. Primary antibodies were incubated at $4{ }^{\circ} \mathrm{C}$ overnight. Secondary antibodies were incubated either for $1 \mathrm{~h}$ (whole mounts) or $2 \mathrm{~h}$ (vibratome sections) at room temperature in the dark. F-actin, abundant in hair cell stereocilia, was labelled with $10 \mathrm{nM}$ phalloidin-Atto $647 \mathrm{~N}$ (Sigma-Aldrich, Gillingham, UK) and nuclei were visualized with $1 \mu \mathrm{M}$ DAPI. Imaging was performed with a Zeiss LSM 510 or 880 confocal microscope using 10x (0.45 N.A.), $20 \times(0.8$ N.A.), and $63 \times$ oil (1.4 N.A.) objectives and Zen 3.0 SR software (Black edition). For image display, any adjustments were made equally to images shown in comparison. During experiments on noise-exposed cochlea there was significantly enhanced labelling of Caprin 1 such that laser excitation power was reduced by half, keeping all other settings the same, to match non-noise exposed tissues.

Ex-vivo cochlear culture explants were immunostained as described previously ${ }^{16}$ and imaged using Zeiss 510NLO multi-photon upright confocal system using a 63x (1.0NA) immersion objective. A minimum of 3 mice per genotype were used and representative images are shown in the figures. Details of antibodies used are in Supplementary Methods. The Caprin1 antibody used was raised against amino acids 356-709, a region encoded by exons $10-19$.

Hair cell counts and IHC-SGN synapse analysis. Maximum intensity projections were generated from confocal Z-stacks $(0.5 \mu \mathrm{m}$ plane intervals, $63 \times$ objective $)$. Z-stack were collected from the $24 \mathrm{kHz}$ region according to the mouse frequency-place map ${ }^{56}$. For hair cell counts, data were quantified in $112 \mu \mathrm{m}$ lengths of the cochlea (see Fig. 3a); Myosin7a and phalloidin were used to identify hair cells, and counted using the Cell-Counter plugin (NIH, Image J v1.52d). Data presented as IHCs or OHCs per $100 \mu \mathrm{m}$ length of the cochlea.

For analysis of the IHC-SGN synapses, a $40.3 \times 21.3 \mu \mathrm{m}$ region of interest (ROI) of the IHC region captured five individual IHCs. ROIs were imported using the Bioformats Importer plugin, splitting them into four individual channels: CtBP2; GluA2; Myosin7a and phalloidin. CtBP2-positive puncta were selected using the threshold tool (with MaxEntropy pre-set function) and total counts and cross-sectional area measurements were made using the Analyze Particles function. The number of CtBP2-positive puncta are presented as numbers per IHC. The same process was applied to measure the GluA2-positive puncta. Use of the maximum projection image for quantification was validated by cross-checking data quantified on a slice-by-slice basis from two wildtype and two Caprin $1^{\text {tm } 3 d / t m 3 d}$ samples.

Scanning electron microscopy (SEM). SEM preparation of the organ of Corti has been described previously ${ }^{57}$. In brief, cochleae were fixed $(2 \mathrm{~h}, 2.5 \%$ glutaraldehyde. $0.1 \mathrm{M}$ cacodylate buffer, $3 \mathrm{mM} \mathrm{CaCl}$, room temperature) and decalcified ( $48 \mathrm{~h}, 4 \%$ EDTA, $4{ }^{\circ} \mathrm{C}$ ) and the organ of Corti dissected, post-fixed in $\mathrm{OsO}_{4}$ and processed through the thiocarbohydrazide-Os-repeated procedure ${ }^{58}$. Processed samples were dehydrated in a graded ethanol series, critical point-dried and sputter coated with platinum. Samples were examined in a JEOL $6700 \mathrm{~F}$ SEM operating at $5 \mathrm{kV}$ by secondary electron detection. Imaging was carried out using SEM Supporter software (System In Frontier, Japan). 
Auditory brainstem response. Repeated ABR recordings were measured in Caprin $1^{+/+}$, Caprin1 $1^{+/ t m 3 d}$ and Caprin $1^{\text {tm3d/tm3d }}$ mice at P28 (P27-29), P56 (P56-57), P98 (P98-101) and P210 (P210-213). Two Caprin1 ${ }^{\text {tm3d/tm3d }}$ mice died before the final ABR measurement at P210. Mice were anaesthetised with ketamine (100 mg/kg, i.p., Ketaset) and xylazine (10 mg/kg, i.p., Rompun). Subcutaneous needle electrodes were inserted on the vertex (active) and over the left (reference) and right (ground) bullae. Free-field click (0.01-ms duration) and tone pip stimuli were presented at $3,6,12,18,24,30,36$ and $42 \mathrm{kHz}$ (5-ms duration, 1-ms rise/fall time) over a range of intensities from 0 to $95 \mathrm{~dB}$ sound pressure level (SPL, $5 \mathrm{~dB}$ steps) as described previously and thresholds were defined by the lowest sound intensity giving a visually-detectable ABR response ${ }^{59}$. Caprin $1^{\text {tm3d/tm3d }}$ mice and wildtype controls, bred and maintained at the UCL EI, underwent ABR recordings at P28 to confirm the auditory phenotype prior to use of the cochleae tissue in immunofluorescence investigations. Click and tone pip stimuli $(8,12,24,32$ and $40 \mathrm{kHz})$ were presented from 0-85 dB SPL in $5 \mathrm{~dB}$ steps using TDT System 3 equipment and software (Tucker-Davis Tech., USA) as described previously ${ }^{60}$. The same ABR protocol was used for the noise exposure experiments. Mice were recovered between successive recordings with atipamezole (1 mg/ $\mathrm{kg}$, i.p. Antisedan). No sex-specific differences in hearing thresholds were observed at any time-point therefore, in all investigations we grouped data from both male and female mice. ABR waveforms were analysed further to determine the amplitude and latency of ABR wave I (at P28, $24 \mathrm{kHz}$ ). In three Caprin $1^{t m 3 d / t m 3 d}$ mice no wave 1 was detected; these were not included in the analyses.

Noise exposure. Mice were anesthetized with ketamine (100 mg/kg weight, i.p., Vetalar) and medetomidine $(0.83 \mathrm{mg} / \mathrm{kg}$ weight, i.p., Domitor $)$ and a bolus of buprenorphine $(0.1 \mathrm{mg} / \mathrm{kg}$ weight, s.c., Vetergesic $)$ was administered separately for additional analgesia. Saline $(0.005 \mathrm{ml} / \mathrm{kg}$ weight, s.c.) was administered for hydration and eyes protected with Viscotears ${ }^{\oplus}$. Mice (P30) were exposed to 8-16 kHz octave-band noise, $100 \mathrm{~dB}$ SPL for $2 \mathrm{~h}$ to generate a temporary threshold shift (TTS ${ }^{30,31}$. In separate experiments, mice were exposed to $8-16 \mathrm{kHz}$ octave-band noise, $110 \mathrm{~dB}$ SPL for $3 \mathrm{~h}$ to generate a permanent threshold shift (PTS) ${ }^{32}$. Noise exposure was performed in a custom sound-proof booth with an RX6 processor (Tucker-Davis Technologies, TDT) as described previously ${ }^{31}$. Pedal-reflex and breathing rate were checked every $30 \mathrm{~min}$. Mice were recovered with atipamazole $(1 \mathrm{mg} / \mathrm{kg}$, s.c.). ABRs were performed pre-noise exposure, $24 \mathrm{~h}, 1$ week and 2 weeks post-exposure. Cochleae were harvested after the final ABR recording for use in subsequent immunofluorescence analysis.

Cochlear cultures. Cochlear explants from P3 mice were prepared as described previously ${ }^{17}$. Experiments were performed on explants from the mid-basal cochlear coil. Cochlear explant cultures were allowed to acclimatize overnight before addition of $0.5 \mathrm{mM}$ sodium arsenite $(1 \mathrm{~h})$ to induce oxidative stress as described previously ${ }^{16}$. Following the sodium arsenite treatment paradigm, explants were fixed in 4\% PFA in PBS (30 min at room temperature), and washed 3 times in PBS prior to immunostaining.

Statistics. Sample sizes were chosen based on our experience with comparable mutant mouse experiments s $^{53}$. The unpaired Student t-test was used to compare two independent variables. The paired t-test was used to compare matched samples e.g. on ABRs performed on the same animal pre- and post- noise exposure. For the initial longitudinal hearing study (i.e. the comparison of ABRs across click and tone-pip stimuli at different timepoints), the ABR thresholds were not normally distributed. Therefore, the data were first transformed using the arcsine transformation and then analysed using separate linear models for each stimulus with a compound symmetric covariance structure and restricted maximum likelihood estimation. This allowed the inclusion of all available data, unlike the repeated measures ANOVA, which is deemed void if any data points are missed ${ }^{61,62}$. For each stimulus the double interaction of genotype and age was measured followed by a Bonferroni correction for multiple testing. The arcsine transformation and mixed model linear pairwise comparison were performed with SPSS v25. Other statistical analyses were undertaken in either GraphPad Prism 7.0d or Excel, and a p-value $<0.05$ was considered significant.

Received: 6 July 2021; Accepted: 12 January 2022

Published online: 14 February 2022

\section{References}

1. Grill, B. et al. Activation/division of lymphocytes results in increased levels of cytoplasmic activation/proliferation-associated protein-1: Prototype of a new family of proteins. J. Immunol. 172, 2389-2400. https://doi.org/10.4049/jimmunol.172.4.2389 (2004).

2. Wang, B., David, M. D. \& Schrader, J. W. Absence of caprin-1 results in defects in cellular proliferation. J. Immunol. 175, 4274-4282. https://doi.org/10.4049/jimmunol.175.7.4274 (2005).

3. Solomon, S. et al. Distinct structural features of caprin-1 mediate its interaction with G3BP-1 and its induction of phosphorylation of eukaryotic translation initiation factor 2alpha, entry to cytoplasmic stress granules, and selective interaction with a subset of mRNAs. Mol. Cell. Biol. 27, 2324-2342. https://doi.org/10.1128/MCB.02300-06 (2007).

4. Kedersha, N. et al. G3BP-Caprin1-USP10 complexes mediate stress granule condensation and associate with 40S subunits. J. Cell Biol. 212, 845-860. https://doi.org/10.1083/jcb.201508028 (2016).

5. Shiina, N., Yamaguchi, K. \& Tokunaga, M. RNG105 deficiency impairs the dendritic localization of mRNAs for Na+/K+ ATPase subunit isoforms and leads to the degeneration of neuronal networks. J. Neurosci. 30, 12816-12830. https://doi.org/10.1523/JNEUR OSCI.6386-09.2010 (2010).

6. Nakayama, K. et al. RNG105/caprin1, an RNA granule protein for dendritic mRNA localization, is essential for long-term memory formation. Elife 6, e29677. https://doi.org/10.7554/eLife.29677 (2017).

7. Shiina, N., Shinkura, K. \& Tokunaga, M. A novel RNA-binding protein in neuronal RNA granules: Regulatory machinery for local translation. J. Neurosci. 25, 4420-4434. https://doi.org/10.1523/JNEUROSCI.0382-05.2005 (2005). 
8. Alberti, S., Gladfelter, A. \& Mittag, T. Considerations and challenges in studying liquid-liquid phase separation and biomolecular condensates. Cell 176, 419-434. https://doi.org/10.1016/j.cell.2018.12.035 (2019).

9. Riggs, C. L., Kedersha, N., Ivanov, P. \& Anderson, P. Mammalian stress granules and P bodies at a glance. J. Cell Sci. 133, 2. https:// doi.org/10.1242/jcs.242487 (2020).

10. Kedersha, N. et al. Correction: G3BP-Caprin1-USP10 complexes mediate stress granule condensation and associate with 40S subunits. J. Cell. Biol. https://doi.org/10.1083/jcb.20150802809202019c (2020).

11. Mahboubi, H. \& Stochaj, U. Cytoplasmic stress granules: Dynamic modulators of cell signaling and disease. Biochim. Biophys. Acta Mol. Basis Dis. 1863, 884-895. https://doi.org/10.1016/j.bbadis.2016.12.022 (2017).

12. Buchan, J. R. \& Parker, R. Eukaryotic stress granules: The ins and outs of translation. Mol. Cell. 36, 932-941. https://doi.org/10. 1016/j.molcel.2009.11.020 (2009).

13. Chen, L. \& Liu, B. Relationships between stress granules, oxidative stress, and neurodegenerative diseases. Oxid. Med. Cell Longev. 2017, 1809592. https://doi.org/10.1155/2017/1809592 (2017).

14. Cao, X., Jin, X. \& Liu, B. The involvement of stress granules in aging and aging-associated diseases. Aging Cell 19, e13136. https:// doi.org/10.1111/acel.13136 (2020).

15. Somasekharan, S. P. et al. YB-1 regulates stress granule formation and tumor progression by translationally activating G3BP1. J. Cell. Biol. 208, 913-929. https://doi.org/10.1083/jcb.201411047 (2015).

16. Gonçalves, A. C. et al. Drug-induced stress granule formation protects sensory hair cells in mouse cochlear explants during ototoxicity. Sci. Rep. 9, 12501. https://doi.org/10.1038/s41598-019-48393-w (2019).

17. Towers, E. R., Kelly, J. J., Sud, R., Gale, J. E. \& Dawson, S. J. Caprin-1 is a target of the deafness gene Pou4f3 and is recruited to stress granules in cochlear hair cells in response to ototoxic damage. J. Cell Sci. 124, 1145-1155. https://doi.org/10.1242/jcs.076141 (2011).

18. Poirrier, A.L., Pincemail, J., Van Den Ackerveken, P., Lefebvre, P.P. \& Malgrange, B. Oxidative stress in the cochlea: an update. Curr. Med. Chem. 17, 3591-3604. https://doi.org/10.2174/092986710792927895 (2010)

19. Someya, S. \& Prolla, T.A. Mitochondrial oxidative damage and apoptosis in age-related hearing loss. Mech. Ageing Dev. 131, 480-486. https://doi.org/10.1016/j.mad.2010.04.006 (2010)

20. Ohlemiller, K.K Contributions of mouse models to understanding of age- and noise-related hearing loss. Brain Res. 1091, 89-102. https://doi.org/10.1016/j.brainres.2006.03.017 (2006)

21. Cannizzaro, E., et al. Exposure to ototoxic agents and hearing loss: A review of current knowledge. Hear. Balance Commun. 12, 166-175. https://doi.org/10.3109/21695717.2014.964939 (2014)

22. Bowl, M.R. \& Dawson, S.J. Age-related hearing loss. Cold Spring Harb. Perspect. Med. 9, a033217. https://doi.org/10.1101/cshpe rspect.a033217 (2019)

23. Muller, S. M. et al. Neural crest origin of perivascular mesenchyme in the adult thymus. J. Immunol. 180, 5344-5351. https://doi. org/10.4049/jimmunol.180.8.5344 (2008).

24. Matsuoka, T. et al. Neural crest origins of the neck and shoulder. Nature 436, 347-355. https://doi.org/10.1038/nature03837 (2005).

25. Jewett, D. L., Romano, M. N. \& Williston, J. S. Human auditory evoked potentials: Possible brain stem components detected on the scalp. Science 167, 1517-1518. https://doi.org/10.1126/science.167.3924.1517 (1970).

26. Willott, J. F. Measurement of the auditory brainstem response (ABR) to study auditory sensitivity in mice. Curr. Protoc. Neurosci. 34, 1-12. https://doi.org/10.1002/0471142301.ns0821bs34 (2006).

27. Parkkonen, L., Fujiki, N. \& Makela, J. P. Sources of auditory brainstem responses revisited: Contribution by magnetoencephalography. Hum. Brain Mapp. 30, 1772-1782. https://doi.org/10.1002/hbm.20788 (2009).

28. Khimich, D. et al. Hair cell synaptic ribbons are essential for synchronous auditory signalling. Nature 434, 889-894. https://doi. org/10.1038/nature03418 (2005).

29. Matsubara, A., Laake, J. H., Davanger, S., Usami, S. \& Ottersen, O. P. Organization of AMPA receptor subunits at a glutamate synapse: A quantitative immunogold analysis of hair cell synapses in the rat organ of Corti. J. Neurosci. 16, 4457-4467 (1996).

30. Kujawa, S. G. \& Liberman, M. C. Adding insult to injury: Cochlear nerve degeneration after "temporary" noise-induced hearing loss. J. Neurosci. 29, 14077-14085. https://doi.org/10.1523/JNEUROSCI.2845-09.2009 (2009).

31. Hesse, L. L. et al. Non-monotonic relation between noise exposure severity and neuronal hyperactivity in the auditory midbrain. Front. Neurol. 7, 133. https://doi.org/10.3389/fneur.2016.00133 (2016).

32. Amanipour, R. M. et al. Noise-induced hearing loss in mice: Effects of high and low levels of noise trauma in CBA mice. Conf. Proc. IEEE Eng. Med. Biol. Soc. 2018, 1210-1213. https://doi.org/10.1109/EMBC.2018.8512525 (2018).

33. Wang, Y., Hirose, K. \& Liberman, M. C. Dynamics of noise-induced cellular injury and repair in the mouse cochlea. J. Assoc. Res. Otolaryngol. 3, 248-268. https://doi.org/10.1007/s101620020028 (2002).

34. Kedersha, N. L., Gupta, M., Li, W., Miller, I. \& Anderson, P. RNA-binding proteins TIA-1 and TIAR link the phosphorylation of eIF-2 alpha to the assembly of mammalian stress granules. J. Cell Biol. 147, 1431-1442. https://doi.org/10.1083/jcb.147.7.1431 (1999).

35. Martin, S. et al. Deficiency of G3BP1, the stress granules assembly factor, results in abnormal synaptic plasticity and calcium homeostasis in neurons. J. Neurochem. 125, 175-184. https://doi.org/10.1111/jnc.12189 (2013).

36. Piecyk, M. et al. TIA-1 is a translational silencer that selectively regulates the expression of TNF-alpha. EMBO J. 19, 4154-4163. https://doi.org/10.1093/emboj/19.15.4154 (2000).

37. Kujawa, S. G. \& Liberman, M. C. Synaptopathy in the noise-exposed and aging cochlea: Primary neural degeneration in acquired sensorineural hearing loss. Hear. Res. 330, 191-199. https://doi.org/10.1016/j.heares.2015.02.009 (2015).

38. Liberman, M. C. Noise-induced and age-related hearing loss: new perspectives and potential therapies. F1000Res 6, 927. https:// doi.org/10.12688/f1000research.11310.1 (2017).

39. Wu, P. Z. et al. Primary neural degeneration in the human cochlea: Evidence for hidden hearing loss in the aging ear. Neuroscience 407, 8-20. https://doi.org/10.1016/j.neuroscience.2018.07.053 (2019).

40. Reijntjes, D. O. J. \& Pyott, S. J. The afferent signaling complex: Regulation of type I spiral ganglion neuron responses in the auditory periphery. Hear. Res. 336, 1-16. https://doi.org/10.1016/j.heares.2016.03.011 (2016).

41. Takago, H. \& Oshima-Takago, T. Pre- and postsynaptic ionotropic glutamate receptors in the auditory system of mammals. Hear. Res. 362, 1-13. https://doi.org/10.1016/j.heares.2018.02.007 (2018).

42. Sephton, C. F. \& Yu, G. The function of RNA-binding proteins at the synapse: Implications for neurodegeneration. Cell. Mol. Life Sci. 72, 3621-3635. https://doi.org/10.1007/s00018-015-1943-x (2015).

43. Barclay, M., Constable, R., James, N. R., Thorne, P. R. \& Montgomery, J. M. Reduced sensory stimulation alters the molecular make-up of glutamatergic hair cell synapses in the developing cochlea. Neuroscience 325, 50-62. https://doi.org/10.1016/j.neuro science.2016.03.043 (2016).

44. Schmitz, F., Konigstorfer, A. \& Sudhof, T. C. RIBEYE, a component of synaptic ribbons: A protein's journey through evolution provides insight into synaptic ribbon function. Neuron 28, 857-872. https://doi.org/10.1016/s0896-6273(00)00159-8 (2000).

45. Stankiewicz, T. R., Gray, J. J., Winter, A. N. \& Linseman, D. A. C-terminal binding proteins: Central players in development and disease. Biomol. Concepts 5, 489-511. https://doi.org/10.1515/bmc-2014-0027 (2014).

46. Wells, H. R. R. et al. GWAS identifies 44 independent associated genomic loci for self-reported adult hearing difficulty in UK biobank. Am. J. Hum. Genet. 105, 788-802. https://doi.org/10.1016/j.ajhg.2019.09.008 (2019). 
47. Keithley, E. M. Pathology and mechanisms of cochlear aging. J. Neurosci. Res. 98, 1674-1684. https://doi.org/10.1002/jnr.24439 (2020).

48. Schaette, R. \& McAlpine, D. Tinnitus with a normal audiogram: Physiological evidence for hidden hearing loss and computational model. J. Neurosci. 31, 13452-13457. https://doi.org/10.1523/JNEUROSCI.2156-11.2011 (2011).

49. Khong, A. et al. The stress granule transcriptome reveals principles of mRNA accumulation in stress granules. Mol. Cell 68, 808-820. https://doi.org/10.1016/j.molcel.2017.10.015 (2017).

50. Tian, S., Curnutte, H. A. \& Trcek, T. RNA granules: A view from the RNA perspective. Molecules 25, 3130. https://doi.org/10. $3390 /$ molecules $25143130(2020)$.

51. Skarnes, W. C. et al. A conditional knockout resource for the genome-wide study of mouse gene function. Nature 474, 337-342. https://doi.org/10.1038/nature10163 (2011).

52. White, J. K. et al. Genome-wide generation and systematic phenotyping of knockout mice reveals new roles for many genes. Cell 154, 452-464. https://doi.org/10.1016/j.cell.2013.06.022 (2013).

53. Chen, J. et al. Spinster homolog 2 (spns2) deficiency causes early onset progressive hearing loss. PLoS Genet. 10, e1004688. https:// doi.org/10.1371/journal.pgen.1004688 (2014).

54. Crispino, G. et al. BAAV mediated GJB2 gene transfer restores gap junction coupling in cochlear organotypic cultures from deaf Cx26Sox10Cre mice. PLoS ONE 6, e23279. https://doi.org/10.1371/journal.pone.0023279 (2011)

55. Eaton-Peabody Laboratories. Video Tutorial, Cochlear Dissection for Whole Mount. Massachusetts Eye and Ear. https://masse yeandear.org/research/otolaryngology/eaton-peabody-laboratories/histology-core. (2017).

56. Muller, M., von Hunerbein, K., Hoidis, S. \& Smolders, J. W. A physiological place-frequency map of the cochlea in the CBA/J mouse. Hear. Res. 202, 63-73. https://doi.org/10.1016/j.heares.2004.08.011 (2005).

57. Bullen, A. et al. Ultrastructural defects in stereocilia and tectorial membrane in aging mouse and human cochleae. J. Neurosci. Res. 98, 1745-1763. https://doi.org/10.1002/jnr.24556 (2020).

58. Davies, S. \& Forge, A. Preparation of the mammalian organ of Corti for scanning electron microscopy. J. Microsc. 147, 89-101. https://doi.org/10.1111/j.1365-2818.1987.tb02821.x (1987).

59. Ingham, N. J. et al. Mouse screen reveals multiple new genes underlying mouse and human hearing loss. PLoS Biol. 17, e3000194. https://doi.org/10.1371/journal.pbio.3000194 (2019).

60. Mistry, N., Nolan, L. S., Saeed, S. R., Forge, A. \& Taylor, R. R. Cochlear implantation in the mouse via the round window: Effects of array insertion. Hear. Res. 312, 81-90. https://doi.org/10.1016/j.heares.2014.03.005 (2014).

61. Duricki, D. A., Soleman, S. \& Moon, L. D. Analysis of longitudinal data from animals with missing values using SPSS. Nat. Protoc. 11, 1112-1129. https://doi.org/10.1038/nprot.2016.048 (2016).

62. Martelletti, E. et al. Synaptojanin2 mutation causes progressive high-frequency hearing loss in mice. Front. Cell Neurosci. 14, 561857. https://doi.org/10.3389/fncel.2020.561857 (2020).

\title{
Acknowledgements
}

We thank Lorenzo Preite for colony management. This work was supported by grants from the MRC [MR/ N004329/1 to SD and JG; G0300212 to KPS] and the Wellcome Trust [091092/Z/09/Z to SD and JG; 100669, 098051 to KPS]. ACG was supported by an Action on Hearing Loss PhD Studentship [EI:595 to JG and SD]

\section{Author contributions}

L.S.N.: acquisition, analysis, visualization, data interpretation, writing-original draft, writing-review and editing. J.C.: acquisition, analysis, visualization. A.-C.G.: acquisition, analysis, visualization. A.B.: acquisition, analysis, visualization. E.R.T.: acquisition, visualization. K.P.S.: supervision, funding acquisition, writing-review and editing. S.J.D.: conceptualization, acquisition, analysis, supervision, funding acquisition, visualization, data interpretation, writing - original draft, writing—review and editing. J.E.G.: conceptualization, acquisition, analysis, supervision, funding acquisition, visualization, data interpretation, writing-original draft, writing-review and editing.

\section{Competing interests}

The authors declare no competing interests.

\section{Additional information}

Supplementary Information The online version contains supplementary material available at https://doi.org/ 10.1038/s41598-022-05657-2.

Correspondence and requests for materials should be addressed to S.J.D. or J.E.G.

Reprints and permissions information is available at www.nature.com/reprints.

Publisher's note Springer Nature remains neutral with regard to jurisdictional claims in published maps and institutional affiliations.

\begin{abstract}
Open Access This article is licensed under a Creative Commons Attribution 4.0 International License, which permits use, sharing, adaptation, distribution and reproduction in any medium or format, as long as you give appropriate credit to the original author(s) and the source, provide a link to the Creative Commons licence, and indicate if changes were made. The images or other third party material in this article are included in the article's Creative Commons licence, unless indicated otherwise in a credit line to the material. If material is not included in the article's Creative Commons licence and your intended use is not permitted by statutory regulation or exceeds the permitted use, you will need to obtain permission directly from the copyright holder. To view a copy of this licence, visit http://creativecommons.org/licenses/by/4.0/.
\end{abstract}

(c) The Author(s) 2022 